\title{
Cascaded integrator comb filters with smoothly varying coefficients for reduced delay in synchrotron feedback loops
}

\author{
A. Schnase, M. Nomura, F. Tamura, and M. Yamamoto \\ JAEA, 2-4 Shirakata-Shirane, Tokai, Ibaraki, 319-1195, Japan \\ S. Anami, E. Ezura, K. Hara, C. Ohmori, A. Takagi, and M. Yoshii \\ High Energy Accelerator Research Organization, 1-1 Oho, Tsukuba, 305-0801, Japan \\ (Received 28 October 2005; published 8 December 2005)
}

\begin{abstract}
The Rapid Cycling Synchrotron (RCS) of the J-PARC complex in Tokai, Japan, is designed to accelerate a high intensity proton beam from $181 \mathrm{MeV}$, and later $400 \mathrm{MeV}$ to $3 \mathrm{GeV}$ in $20 \mathrm{~ms}$ within the $40 \mathrm{~ms}$ machine cycle. The beam power up to $1 \mathrm{MW}$ demands a stable beam control to avoid excessive losses and activation of the accelerator chain. The fully digital control system is based on quadrature modulation and demodulation. In the amplitude control loops standard FIR filters separate the harmonics $(h=2)$ and $(h=4)$ after down conversion. For the phase loops at $(h=2)$ and $(h=4)$, intended to damp synchrotron oscillations, the delay in a FIR filter would limit the loop stability. Cascaded integrator comb filters, also called CIC filters, provide a shorter delay because they filter the longitudinal beam signal only where it is necessary. The notches are located at multiples of the revolution frequency of the proton beam. For fixed frequency accelerator applications, digital comb filters with fixed clock frequency are widely used to improve loop stability. For variable frequency accelerator applications, as in a proton synchrotron, where the frequency swing is larger than the notch width, usually the clock frequency of the comb filter is variable and chosen to be an integer multiple of the particle revolution frequency. At J-PARC RCS, the clock frequency has to be fixed. Tracking the frequency would require a variable noninteger number of filter taps. Here we present a filter, based on the weighted output of 2 CIC filters with variable length, and one tap difference. The filter function looks like a CIC with smoothly varying coefficients, where the notches follow the revolution frequency of the proton beam. The delay of this filter is approximately half of the corresponding FIR filter, so that the phase loops have a higher stability margin.
\end{abstract}

DOI: 10.1103/PhysRevSTAB.8.122001

PACS numbers: 29.20.Lq, 07.50.Qx, 84.30.Vn

\section{INTRODUCTION}

The Rapid Cycling Synchrotron (RCS) of the J-PARC complex [1] in Tokai, Japan, is designed to accelerate a high intensity proton beam from $181 \mathrm{MeV}$ in a first stage, and $400 \mathrm{MeV}$ in a later stage to $3 \mathrm{GeV}$ in $20 \mathrm{~ms}$ at $25 \mathrm{~Hz}$ repetition frequency. The beam power will be up to $1 \mathrm{MW}$; therefore it is mandatory to keep the beam stable to avoid excessive losses and activation of the accelerator chain.

The acceleration cavities for RCS [2] are loaded with magnetic alloy, adjusted so that their quality factor is approximately 2, which allows dual harmonic operation. These cavities require no tuning loop in contrast to ferrite cavities.

Dual harmonic operation [3] uses $(h=2)$ for acceleration and $(h=4)$ for longitudinal beam shaping. For precise control and synchronism of the amplitudes and phases of the 11 cavities, the fully digital control system [4] uses a common reference oscillator to supply a phase reference for both harmonics to the cavity driver modules. The signal processing is based on digital quadrature modulation and demodulation. For the amplitude control loops [5] standard FIR filters [6] are used to separate the harmonics. For the phase loops at $(h=2)$ and $(h=4)$, which damp synchrotron oscillations, the delay in a FIR filter would limit the loop stability.

Digital cascaded-integrator-comb filters, also called CIC filters have a shorter delay, because they filter the signal, just where it is necessary, e.g., the notches of the filter are located at multiples of the revolution frequency of the proton beam. Originally, CIC filters were introduced for audio applications [7]. A reference for the application of digital comb filters in a high-energy proton synchrotron is given in [8]. For variable frequency accelerator applications, as in a proton synchrotron, where the frequency swing is larger than the notch width, the clock frequency of the comb filter is an integer multiple of the particle revolution frequency [9], so that a one-turn delay is achieved. For fixed frequency accelerator applications, as in electron or positron synchrotron storage rings [10], digital comb filters $[11,12]$ with fixed clock frequency are widely used to improve the loop stability.

At J-PARC RCS, the clock frequency is fixed to a common $12 \mathrm{MHz}$ reference. The filter presented here is based on the weighted output of 2 CIC filters with variable length, and one tap difference. The filter function looks like a CIC with smoothly varying coefficients, where the 
notches follow the revolution frequency of the proton beam.

\section{DERIVING A CIC FILTER WITH VARIABLE LENGTH}

\section{A. Digital receiver basics}

The basic subsystem for digital down conversion of a revolution harmonic is shown in Fig. 1. The longitudinal beam signal is detected by a fast current transformer (FCT), filtered by an analog low pass, and sampled by an AD converter. A digital local oscillator with sine and cosine of the selected center frequency multiplies the digital data. The digital low-pass filters remove the upper sidebands after mixing. Then a digital coordinate transformer [13] processes the in-phase and quadrature components of the detected signal, so that amplitude and phase appear at the outputs. The components, such a digital receiver is made of, are described in [14].

The digital filter after down conversion of the selected harmonic has a low-pass characteristic, which rejects unwanted signals. In a synchrotron we expect to have longitudinal signals at each revolution frequency harmonic. Therefore the cutoff frequency of the filters has to be smaller than the revolution frequency. At injection time of RCS, at $181 \mathrm{MeV}$, the revolution frequency is $f_{\text {rev }}=$ $469250 \mathrm{~Hz}$. At extraction time, at $3 \mathrm{GeV}$, the revolution frequency is $835867 \mathrm{~Hz}$.

If we were allowed to use a variable system clock, we could select a fixed number of taps $n_{\text {tap }}$ and then apply a variable clock frequency $f_{\text {clk }}$, which tracks the revolution frequency $f_{\text {rev }}$ of the synchrotron.

$$
f_{\text {clk }}=n_{\text {tap }} \cdot f_{\text {rev }}
$$

This means for a CIC filter, which is a special type of FIR filter where all taps have the same weight, that the notch spacing will follow the acceleration in the synchrotron.

\section{B. Standard CIC filters}

An example of such filter, shown in Fig. 2 has 32 taps. The clock frequency for this filter would vary from $15.016 \mathrm{MHz}$ to approximately $26.7477 \mathrm{MHz}$ during the RCS cycle. Such type of filtering is standard for the lowlevel system of COSY [15], with a revolution frequency between 490 and $1578 \mathrm{kHz}$. At COSY the number of taps is set to 16 , so that the maximum clock frequency is not much higher than $25 \mathrm{MHz}$. This sample rate limit was defined by the available 12-bit AD-converters and 16-bit multipliers around 1995.

The transfer function for a CIC filter with $n_{\text {tap }}$ taps is

$$
h(f)=\frac{\sin \left(n_{\text {tap }} \pi \frac{f}{f_{\text {clk }}}\right)}{n_{\text {tap }} \cdot \sin \left(\pi \frac{f}{f_{\text {clk }}}\right)} \exp \left(-j n_{\text {tap }} \pi \frac{f}{f_{\text {clk }}}\right) .
$$

With $\Omega=f / f_{\text {clk }}$, it becomes

$$
h(\Omega)=\frac{\sin \left(n_{\mathrm{tap}} \pi \Omega\right)}{n_{\mathrm{tap}} \cdot \sin (\pi \Omega)} e^{-j n_{\mathrm{tap}} \pi \Omega} .
$$

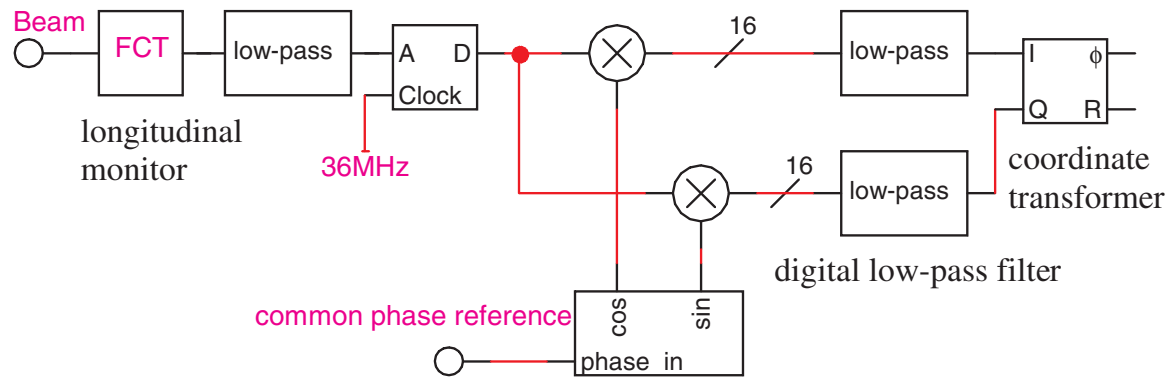

FIG. 1. (Color) Digital receiver for harmonic down conversion with $I / Q$ demodulation.

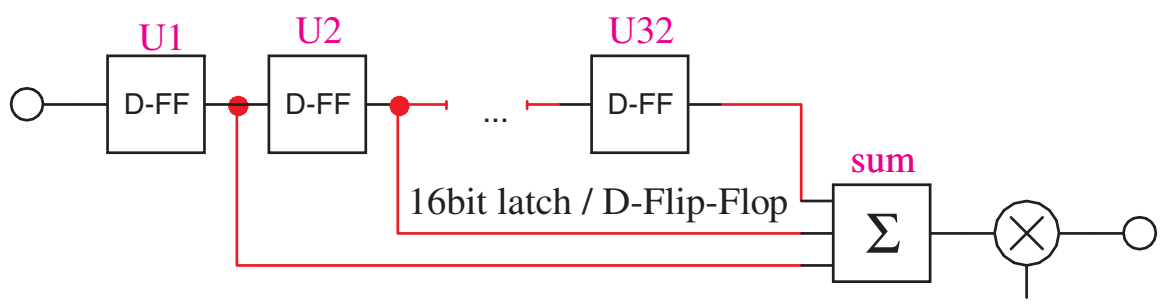

scale by $1 / 32$

FIG. 2. (Color) CIC filter for a variable clock with fixed number of 32 taps. 


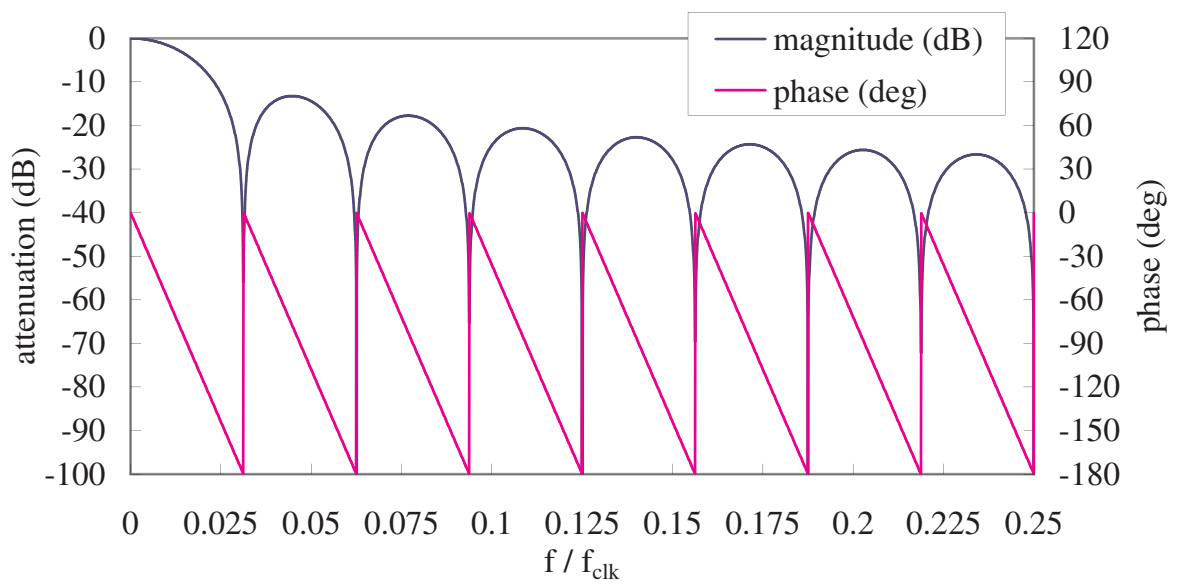

FIG. 3. (Color) Transfer function of a CIC filter with 32 taps for variable clock.

$$
\begin{aligned}
|h(\Omega)| & =\operatorname{abs}\left(\frac{\sin \left(n_{\text {tap }} \pi \Omega\right)}{n_{\text {tap }} \cdot \sin (\pi \Omega)}\right) ; \\
\theta(\Omega) & =-n_{\text {tap }} \pi \Omega+\pi \operatorname{int}\left(n_{\text {tap }} \Omega\right) .
\end{aligned}
$$

The transfer function for the filter with 32 taps is plotted in Fig. 3. The first zero of the transfer function is at $f / f_{\text {clk }}=$ $1 / 32=0.03125$. All harmonics of the revolution frequency $f_{\text {rev }}$ below $f_{\text {clk }}$ are attenuated.

However, this solution is not applicable for J-PARC RCS, because the clock frequency of the digital system for RCS is fixed to $36 \mathrm{MHz}$. In J-PARC, the injector Linac, the RCS, and the main ring (MR) are synchronized to a common $12 \mathrm{MHz}$ reference oscillator [16]. With fixed clock frequency, there is the other option to change the number of taps as a function of revolution frequency during RCS acceleration. However, this results in another conflict. The frequency pattern of the RCS cycle is a smooth function, and one expects the filter notches to track accordingly. However, the number of taps of a simple CIC filter can only be changed in integer steps, so that the resulting frequency step for the notches is bigger than the notch width. In other words, there is no straightforward version of a digital delay for a fraction of a clock cycle. This restriction is removed by linear interpolation between 2 CIC filters, which have a different number of filter taps, as is shown in Fig. 4, and then explained.

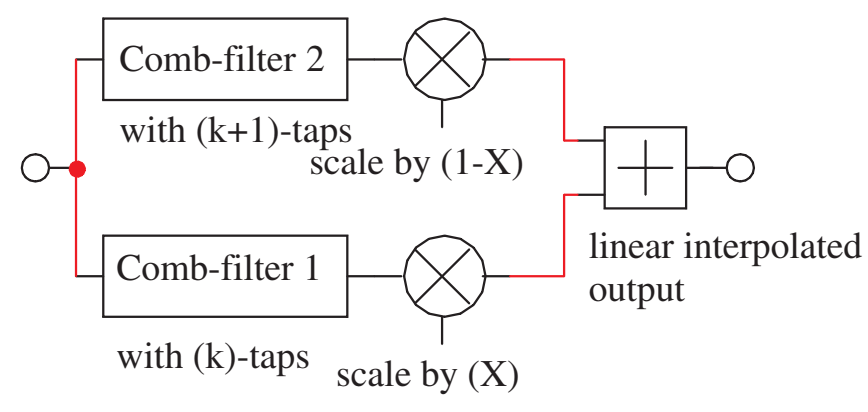

FIG. 4. (Color) Interpolation between 2 comb filters.
At time of injection at RCS, the optimum CIC filters for a variable clock could have 76 taps at a clock frequency of $35.663 \mathrm{MHz}$ [Fig. 5(a)] or 77 taps at a clock frequency of $36.13225 \mathrm{MHz}$ [Fig. 5(b)]. For a fixed clock of $36 \mathrm{MHz}$, the number of taps would be the fractional number 76.718 in between. The magnitude of the transfer functions of the filters in Figs. 5(a) and 5(b) that are almost identical, are shown in Fig. 6.

\section{Interpolation at fixed clock frequency}

In contrast to Figs. 5(a) and 5(b), here we assume, that both filters, the one with 76 taps, and the other with 77 taps are running at a fixed clock of $36 \mathrm{MHz}$. The transfer functions of both CIC filters have different frequencies where maximum attenuation occurs. These notches are at multiples of $(36 \mathrm{MHz} / 77)=467.53 \mathrm{kHz}$ for the filter with 77 taps, and at multiples of $(36 \mathrm{MHz} / 76)=$ $473.68 \mathrm{kHz}$ for the filter with 76 taps. This is shown for the first 2 notches in Fig. 7. The frequency to be suppressed, $f_{\text {rev }}=469.25 \mathrm{kHz}$, is located in between the notches of both filters.
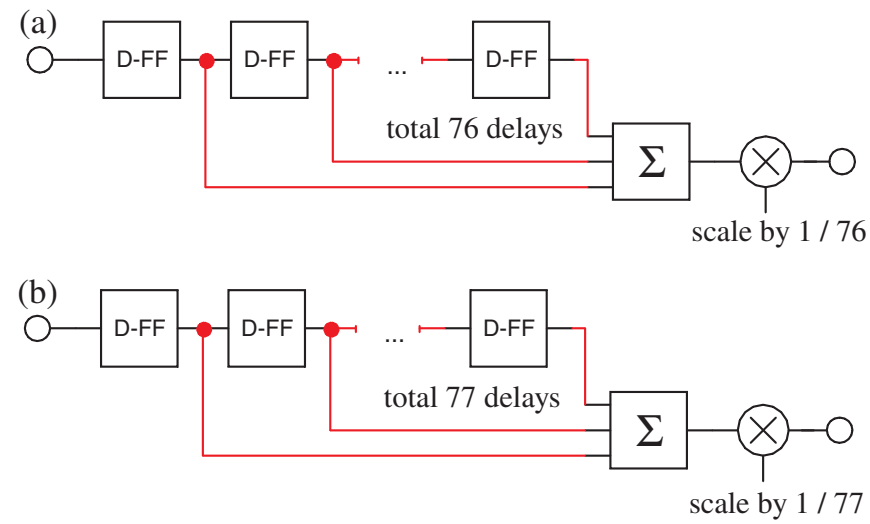

FIG. 5. (Color) (a) CIC filter with 76 taps for RCS injection with $35.663 \mathrm{MHz}$ clock; (b) CIC filter with 77 taps at RCS injection with $36.13225 \mathrm{MHz}$ clock. 


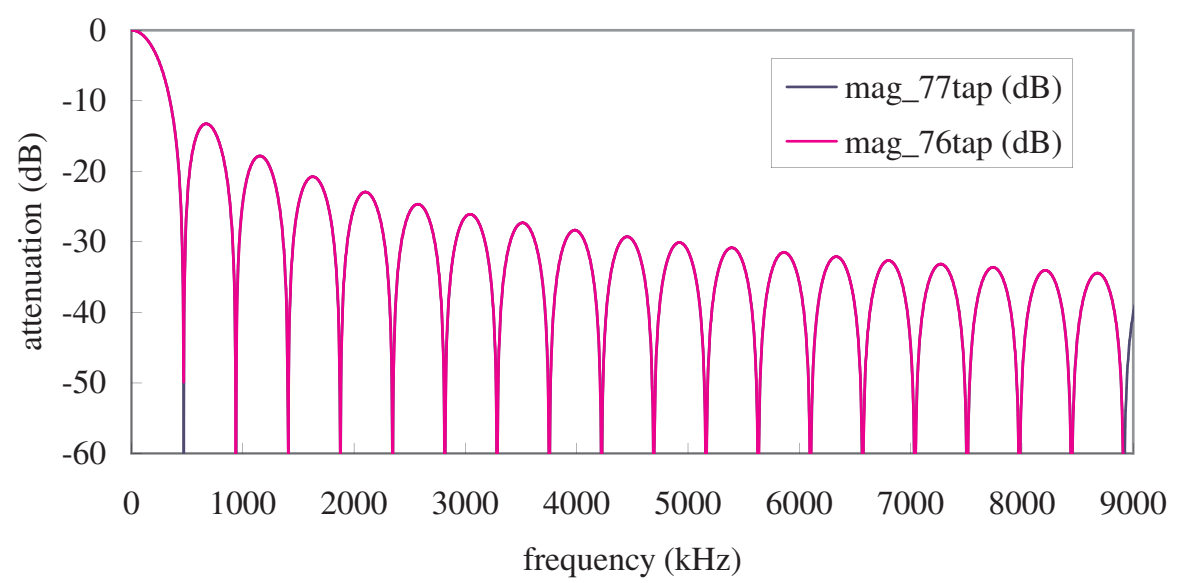

FIG. 6. (Color) Transfer function of CIC filters for variable clock with 76 taps at $76 f_{\text {rev }}$ and 77 taps at $77 f_{\text {rev }}$ at RCS injection with $f_{\text {rev }}=469.25 \mathrm{kHz}$. The harmonics of $f_{\text {rev }}$ are attenuated.

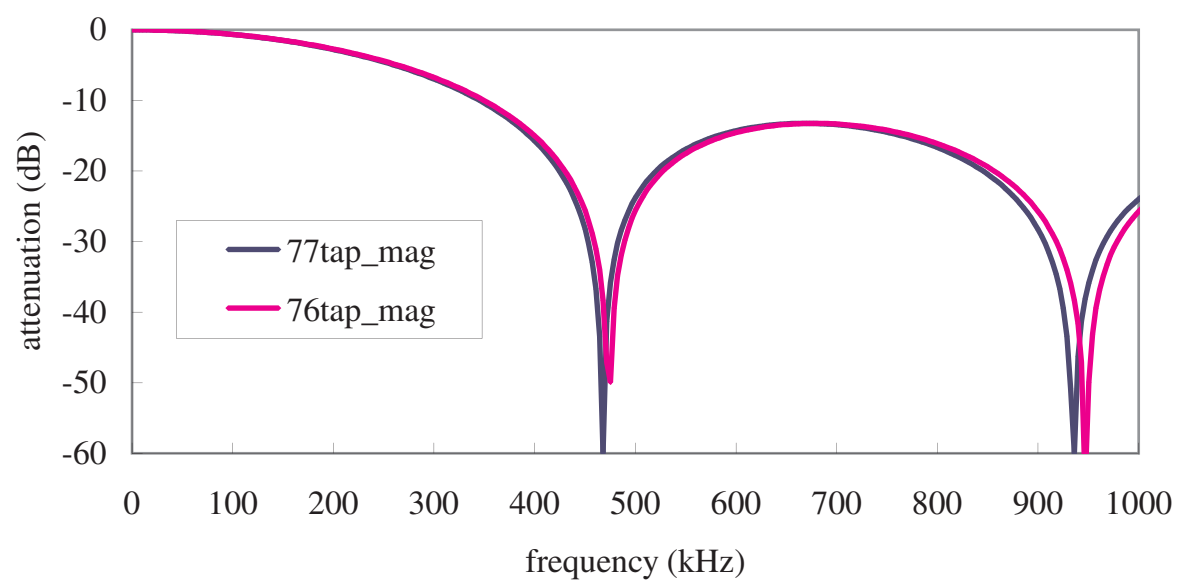

FIG. 7. (Color) CIC filter with 76 and 77 taps: attenuation at $36 \mathrm{MHz}$ fixed clock frequency for $f_{\text {rev }}=469 \mathrm{kHz}$ (RCS injection).

The optimum number of taps $k_{\mathrm{opt}}$ is noninteger:

$$
\begin{aligned}
k_{\mathrm{opt}} & =f_{\mathrm{clk}} / f_{\text {rev }} ; \\
\text { here } k_{\mathrm{opt}} & =36 \mathrm{MHz} / 0.46925 \mathrm{MHz}=76.718 .
\end{aligned}
$$

We define the integer number of taps:

$$
k_{\text {int }}=\operatorname{int}\left(k_{\text {opt }}\right)=\operatorname{int}\left(f_{\text {clk }} / f_{\text {rev }}\right) ; \quad \text { here } k_{\text {int }}=76 \text {, }
$$

and the remaining fraction:

$$
k_{\text {frac }}=k_{\mathrm{opt}}-k_{\mathrm{int}}=k_{\mathrm{opt}}-\operatorname{int}\left(k_{\mathrm{opt}}\right) ; \quad \text { here } k_{\text {frac }}=0.718 .
$$

Then a scaling process, similar to linear interpolation between two points is applied. The output of the filter with 77 taps is scaled by $k_{\text {frac }}=0.718$, and the output of the filter with 76 taps by $\left(1-k_{\text {frac }}\right)=(1-0.718)=0.282$ and both scaled values are added. The combined filter shown in Fig. 8 has a transfer function, plotted in Fig. 9, which is almost like the transfer function of a single CIC filter with variable clock. The D-flip-flop resources for the delays $1 \ldots 76$ can be used for both filters. Also the generation of the sum can be shared. A filter version with optimized usage of resources, important for implementation in FPGA (field programmable gate array), is shown in Fig. 10. The result for the average of 76 taps is available one clock cycle earlier than the result for the 77 tapstherefore another D-flip-flop is put before scaling with $(1 / 76)$. The filter in Fig. 10 is equivalent to a nonsymmetric FIR filter, shown in Fig. 11, where the first 76 coefficients are unity, and the coefficient 77 is variable between 0 and 1 , and finally a scaling is applied to obtain unity gain at dc. At first glance, it looks obvious, to combine the cascaded multipliers to save resources. However, the final scaling for unity gain at dc requires a signal-processing environment that supports floating-point numbers.

\section{Interpolated CIC filters with a variable number of taps}

Between injection and extraction of RCS, the revolution $(h=1)$ frequency pattern will sweep from $469.25 \mathrm{kHz}$ to 


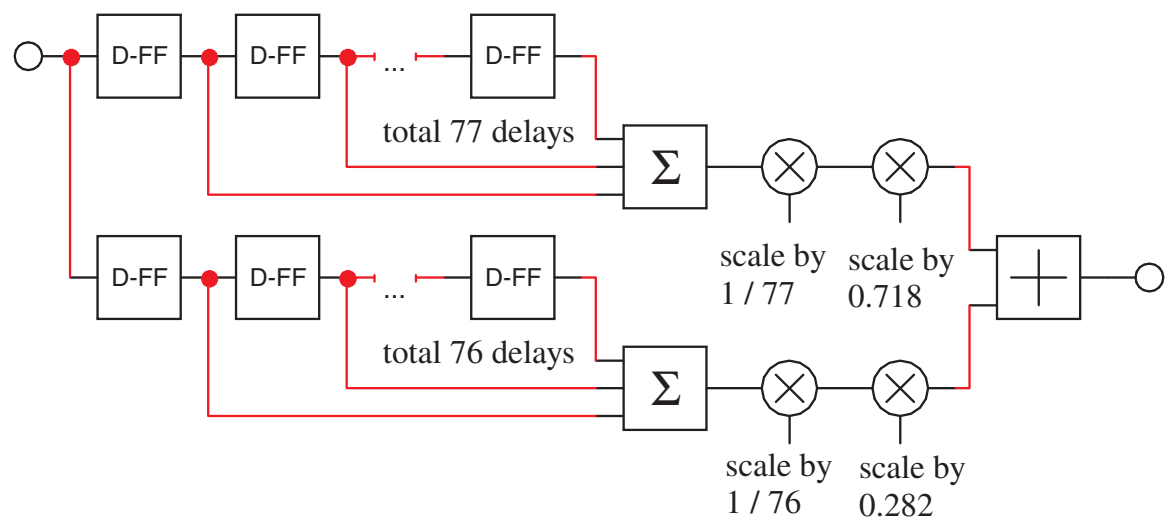

FIG. 8. (Color) Two CIC filters combined for a noninteger number of taps (here 76.718).

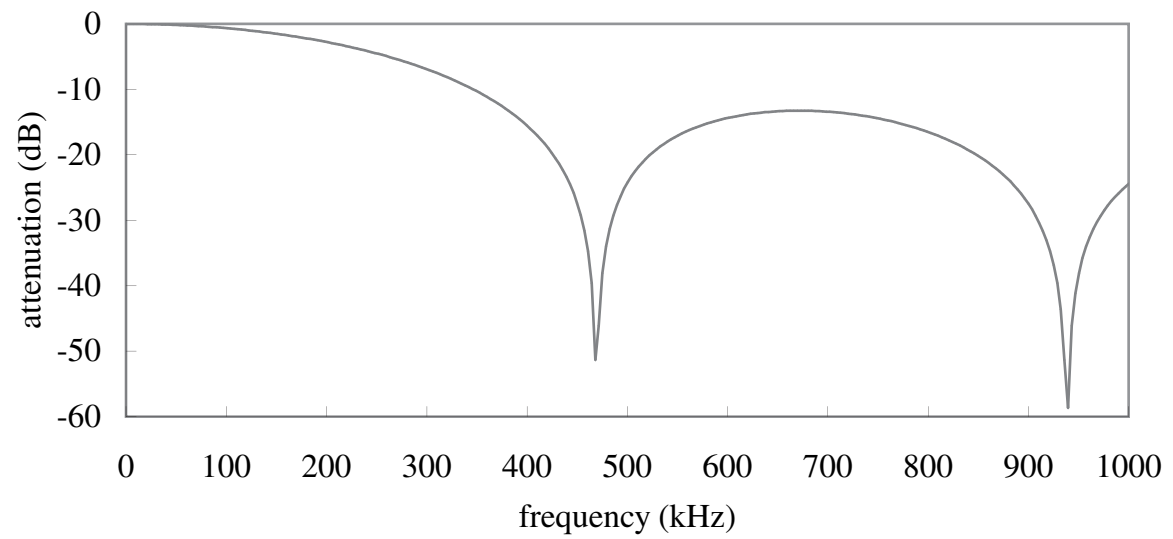

FIG. 9. The transfer function of the two combined CIC filters in Fig. 8: 76 taps and 77 taps at $f_{\text {clk }}=36 \mathrm{MHz}$. The first notch is at $468 \mathrm{kHz}$, the next at $939.6 \mathrm{kHz}$.

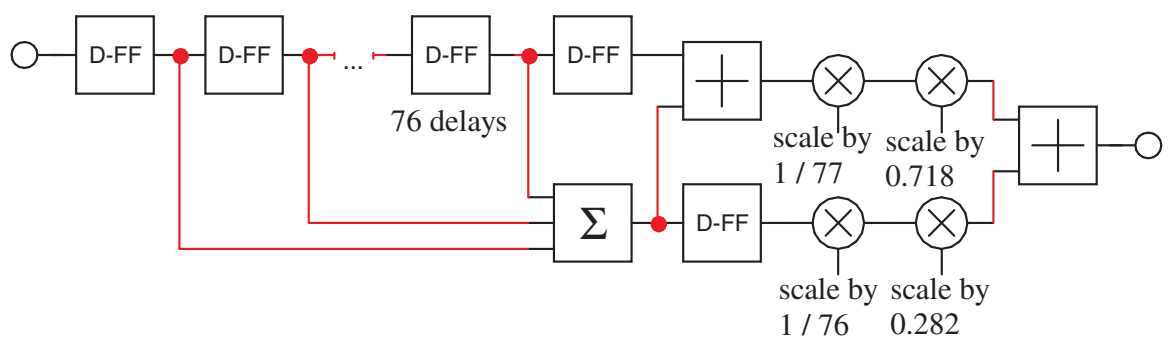

FIG. 10. (Color) Optimized version of 2 combined CIC filters of Fig. 8.

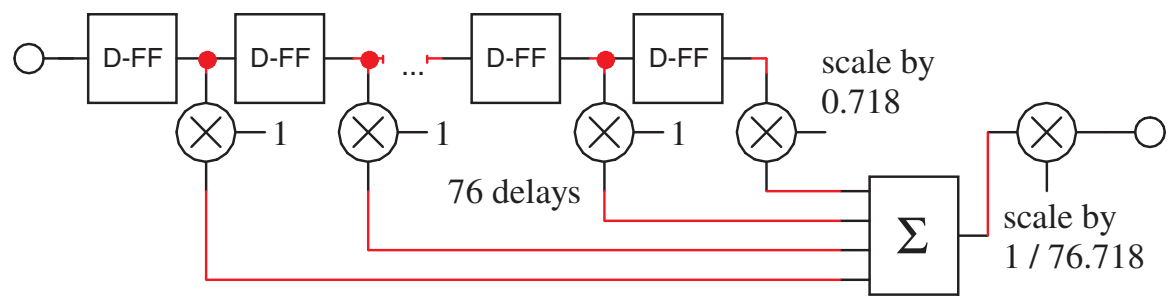

FIG. 11. (Color) FIR equivalent version of Fig. 10 where 76 coefficients are unity, and the last coefficient is variable. 
approximately $835.866 \mathrm{kHz}$. In a later stage, when the injector LINAC will be upgraded to $400 \mathrm{MeV}$, the injection frequency of RCS will rise to $613.690 \mathrm{kHz}$, which is included in the filter operating range. For the expected sweep, the factor between revolution frequency and clock frequency $k_{\text {opt }}=f_{\text {clk }} / f_{\text {rev }}$ decreases from 76.718 at $181 \mathrm{MeV}$ injection to 43.069 at $3 \mathrm{GeV}$ extraction. In case of $400 \mathrm{MeV}$ injection, the factor $k_{\mathrm{opt}}$ varies between 58.662 at injection and 43.069 at extraction. In order to be prepared for changes in the future, and for testing purposes, the factor $k_{\mathrm{opt}}$ is defined with some safety margin. The chosen valid range for $k_{\text {opt }}$ is between 40 and 80 . The delay structure is composed of a fixed delay and a variable delay. For a fixed delay of 32 taps, which is easy to realize in a tree structure, the remaining variable delay will be changeable from 8 to 48 taps. The number of delays $k_{\text {int }}$ is an integer number, with $k_{\text {int }}=\operatorname{int}\left(k_{\text {opt }}\right)=\operatorname{int}\left(f_{\text {clk }} / f_{\text {rev }}\right)$ from Eq. (6). The corresponding circuit is shown in Fig. 12.

The function is explained by an example. The revolution frequency is assumed to be $f_{\text {rev }}=816.326 \mathrm{kHz}$. Then $k_{\text {opt }}=36 \mathrm{MHz} / 0.816326 \mathrm{MHz}=44.1, \quad k_{\text {int }}=44$, and $k_{\text {frac }}=0.1$. The fixed delay of 32 taps and the variable delay of 12 taps result in a total of $k_{\text {int }}=44$ delays. As $k_{\text {opt }}$ is nearer to 44 than 45 , the interpolated CIC-filter transfer function is expected to be close to the transfer function of a single CIC filter with 44 taps. In the upper signal path, the sum of 45 taps is scaled by $(1 / 45)$. In the next multiplier, it is scaled by $\left(k_{\text {frac }}\right)=0.1$.

In the lower signal path, the sum of 44 taps is first scaled by $(1 / 44)$. In the next multiplier, it is scaled by $(1-$ $\left.k_{\text {frac }}\right)=1-0.1=0.9$.

\section{IMPLEMENTATION DETAILS OF THE INTERPOLATED CIC FILTER}

\section{A. Scaling factors $\left(\boldsymbol{k}_{\text {frac }}\right)$ and $\left(1-k_{\text {frac }}\right)$}

For implementation of the filter, the scaling factors have a limited definition range. The scale factor $\left(k_{\mathrm{frac}}\right)$ in the upper filter path of Fig. 12 is defined for positive numbers from zero up to, but not including " 1 ". The scale factor $\left(1-k_{\text {frac }}\right)$ in the lower part of the filter is defined for positive numbers slightly bigger than zero (zero is not included) up to including 1. To avoid floating-point operation, fixedpoint arithmetic is implemented, where the scaling factors are stored in a pattern memory.

The sum of these scaling factors $\left(k_{\text {frac }}\right)+\left(1-k_{\text {frac }}\right)$ is always 1 , therefore it is sufficient, to store only one of them in the pattern memory. As a straightforward implementation, the number $k_{\text {opt }}$ is stored as a fixed-point number in a 16-bit wide pattern with 40000 memory locations. This is compatible with ramping function generation for the low-level rf system of RCS [17], where for example the voltage is defined by pattern memories with 40000 entries, so that each microsecond a new value is available during the $40 \mathrm{~ms}$ RCS machine cycle. The upper 6-bit (MSB) contain the integer part $\left(k_{\text {int }}-32\right)$. The value 32 is subtracted for the fixed delay of 32 taps, and to keep the number within 6-bit range. The remainder $k_{\text {frac }}=k_{\text {opt }}-$ $k_{\text {int }}$ is multiplied by 1024 , which results in a 10-bit positive integer number between 0 and 1023 (LSB). Table I gives examples for the pattern as function of frequency. The data format is given in Table II.

The full range of the table for $f_{\text {rev }}$ from 444.445 to $900.000 \mathrm{kHz}$ was checked for consistency. The momentum of RCS will cycle like

$$
p(t)=\frac{p_{\text {top }}+p_{\text {inj }}}{2}-\frac{p_{\text {top }}-p_{\text {inj }}}{2} \cos (2 \cdot \pi \cdot 25 \mathrm{~Hz} \cdot t)
$$

within $20 \mathrm{~ms}$ from $p_{\text {inj }}=610.259 \mathrm{MeV} / c$ to $p_{\text {top }}=$ $3824.87 \mathrm{MeV} / c$, so that the kinetic energy increases from 181 to $3000 \mathrm{MeV}$. Near injection, the lowest bit of the filter pattern will change when the revolution frequency changes by $6 \mathrm{~Hz}$. The first change will occur at $16 \mu \mathrm{s}$. Near extraction, the lowest bit of the filter pattern will change when the revolution frequency changes by $12 \mathrm{~Hz}$. The last change during the $20 \mathrm{~ms}$ acceleration time happens at $19.854 \mathrm{~ms}$. Between 2 and $4 \mathrm{~ms}$, the revolution frequency changes $37 \ldots 49 \mathrm{~Hz}$ per $\mu \mathrm{s}$. Then the pattern value for the CIC filter changes by the value 5 or 6 each microsecond step.

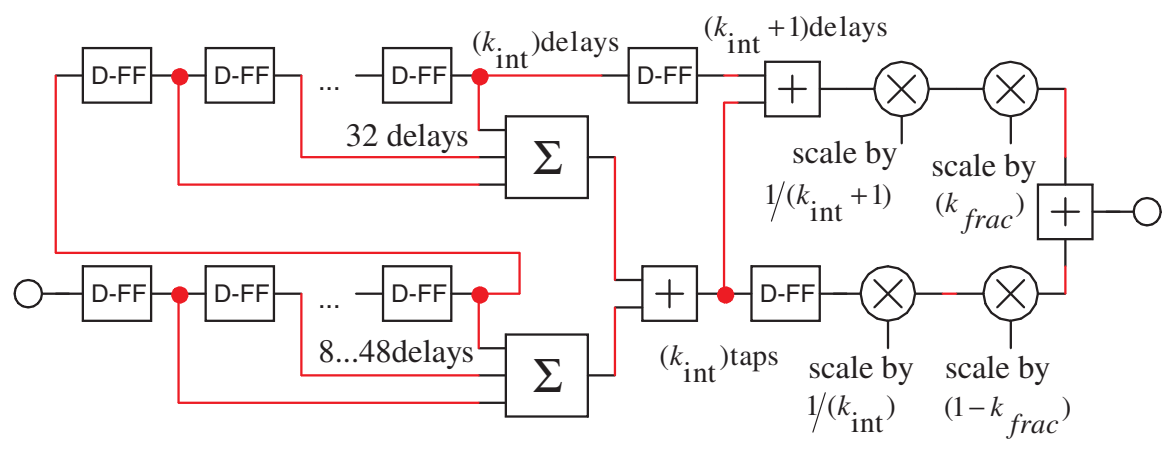

FIG. 12. (Color) The interpolated CIC filter for variable relation $k_{\text {opt }}$ between $36 \mathrm{MHz}$ clock frequency and revolution frequency. 
TABLE I. Examples of the filter pattern for selected frequencies $f_{\text {rev }}$.

\begin{tabular}{llclclccc}
\hline \hline$f_{\text {rev }}(\mathrm{kHz})$ & \multicolumn{1}{c}{$k_{\text {opt }}$} & $k_{\text {int }}$ & $k_{\text {int }}-32$ & $k_{\text {int }}-32(\mathrm{MSB})$ & $k_{\text {frac }}$ & Int(1024 $\left.k_{\text {frac }}\right)$ & Remain (LSB) & Pattern (hex) \\
\hline 444.445 & 80.999899 & 80 & 48 & C000 & 0.999899 & 1023 & $03 \mathrm{FF}$ & C3FF \\
450.000 & 80 & 80 & 48 & C000 & 0.0 & 0 & 0000 & C000 \\
$\mathbf{4 6 9 . 2 5 0}$ & 76.718167 & 76 & 44 & B 000 & 0.718167 & 735 & $02 \mathrm{DF}$ & B2DF \\
500.000 & 72 & 72 & 40 & A000 & 0.0 & 0 & 0000 & A000 \\
$\mathbf{6 1 3 . 6 9 1}$ & 58.66144 & 58 & 26 & 6800 & 0.66144 & 677 & $02 \mathrm{~A} 5$ & $6 \mathrm{AA5}$ \\
750.000 & 48 & 48 & 16 & 4000 & 0.0 & 0 & 0000 & 4000 \\
$\mathbf{8 3 5 . 8 6 7}$ & 43.06905 & 43 & 11 & $2 \mathrm{C} 00$ & 0.06905 & 70 & 0046 & $2 \mathrm{C} 46$ \\
900.000 & 40 & 40 & 8 & 2000 & 0.0 & 0 & 0000 & 2000 \\
\hline \hline
\end{tabular}

TABLE II. The data format of the 16-bit filter pattern.

\begin{tabular}{lc}
\hline \hline Upper 6-bit (MSB) & Lower 10-bit (LSB) \\
\hline$k_{\text {int }}-32$ & $\operatorname{Int}(1024 \mathrm{kfrac})$ \\
\hline \hline
\end{tabular}

The connection of the pattern to the filter is shown in Fig. 13. Brackets "[]" indicate the word length. The 6-bit value $\left(k_{\text {int }}-32\right)$ selects the number of delays. The 10-bit value $\operatorname{Int}\left(1024 k_{\text {frac }}\right)$ is for the scaling multiplier in the upper path of Fig. 12. The sign bit [bit 15] of this multiplier input is set to zero. [Bit 14 ... bit 5] of the multiplier input are connected to the 10-bit from the pattern. [Bit $4 \ldots$ bit 0 ] of the 16-bit multiplier are set to zero. For the scaling multiplier in the lower path of Fig. 12, $\left(1-k_{\text {frac }}\right)$ is computed by subtracting the scaled number int $\left(1024 k_{\text {frac }}\right)$ from 32768 . The value of 32768 at the $A$ input of the subtracter $(A-B)$ in Fig. 13 is a 17-bit number in 2's complement. Therefore the multiplier for scaling needs a 17-bit input. Alternatively, the number could be changed to 32767 , because the resulting error is small, so that a 16-bit input is sufficient.

\section{B. Scaling with the number of taps}

The circuit shown in Fig. 12 contains one multiplier to scale by $1 /\left(k_{\text {int }}+1\right)$ and another to scale by $1 /\left(k_{\text {int }}\right)$. This is shown in detail in Fig. 14. For digital signal processing with integer arithmetic, one tries to avoid division opera-

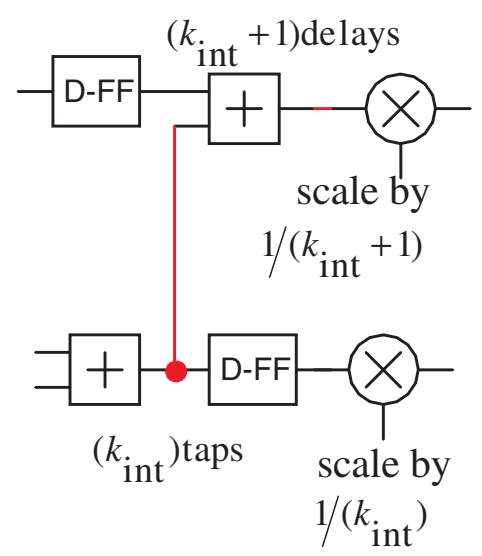

FIG. 14. (Color) Filter scaling to dc unity gain by integer number of taps (detail of Fig. 12).

tions. As the number of integer values for $k_{\text {int }}$ is limited to the interval $[40,80]$ in case of RCS frequency range, we use a table containing approximate reciprocal values. The approximate values are calculated by dividing the 22-bit value $2^{22}=4194304$ by $k_{\text {int }}$. After rounding to the next integer, an 18-bit positive 2's complement number is obtained. Table III lists the numbers. For the multiplier output, finally a scaling with a 22-bit-shift of $2^{22}=4194304$ is necessary, which is implemented by selected the appropriate multiplier outputs. Finally, 3 CIC filters as shown in Fig. 12 will be cascaded. Therefore the scaling table has

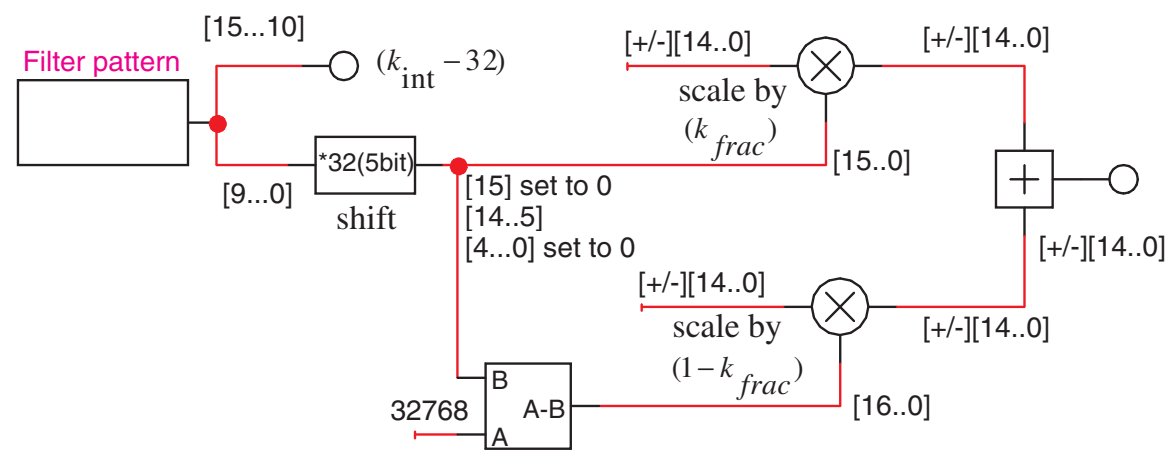

FIG. 13. (Color) The data flow from memory pattern to filter (a detail to Fig. 12). 
TABLE III. Selected values of the table for the scaling factor $\left(1 / k_{\text {int }}\right)$.

\begin{tabular}{lccc}
\hline \hline$k_{\text {int }}$ & $2^{22} / k_{\text {int }}$ & 18-bit signed & Hex \\
\hline 40 & 104857.6 & 104858 & $1999 A$ \\
43 & 97541.95349 & 97542 & $17 D 06$ \\
58 & 72315.58621 & 72316 & $11 \mathrm{~A} 7 \mathrm{C}$ \\
76 & 55188.21053 & 55188 & 0D794 \\
80 & 52428.8 & 52429 & 0CCCD \\
\hline \hline
\end{tabular}

several "read" outputs. For addressing the table, we use $\left(k_{\text {int }}-32\right)$, which is defined between 8 and 48 .

\section{The structure of the variable delay}

The variable delay generates the 22-bit wide sum of $8 \ldots 48$ samples. The number of samples is defined by
TABLE IV. Explanation of the selection switches in Fig. 15.

\begin{tabular}{lc}
\hline \hline$k_{\text {int }}-32$ & Switch matrix condition \\
\hline 48 & All switches S9 to S48 are closed \\
47 & Switches S9 to S47 are closed. S48 is open \\
$\ldots$ & $\ldots$ \\
10 & S9 and S10 are closed \\
9 & Only S9 is closed \\
8 & None of the switches are closed \\
\hline \hline
\end{tabular}

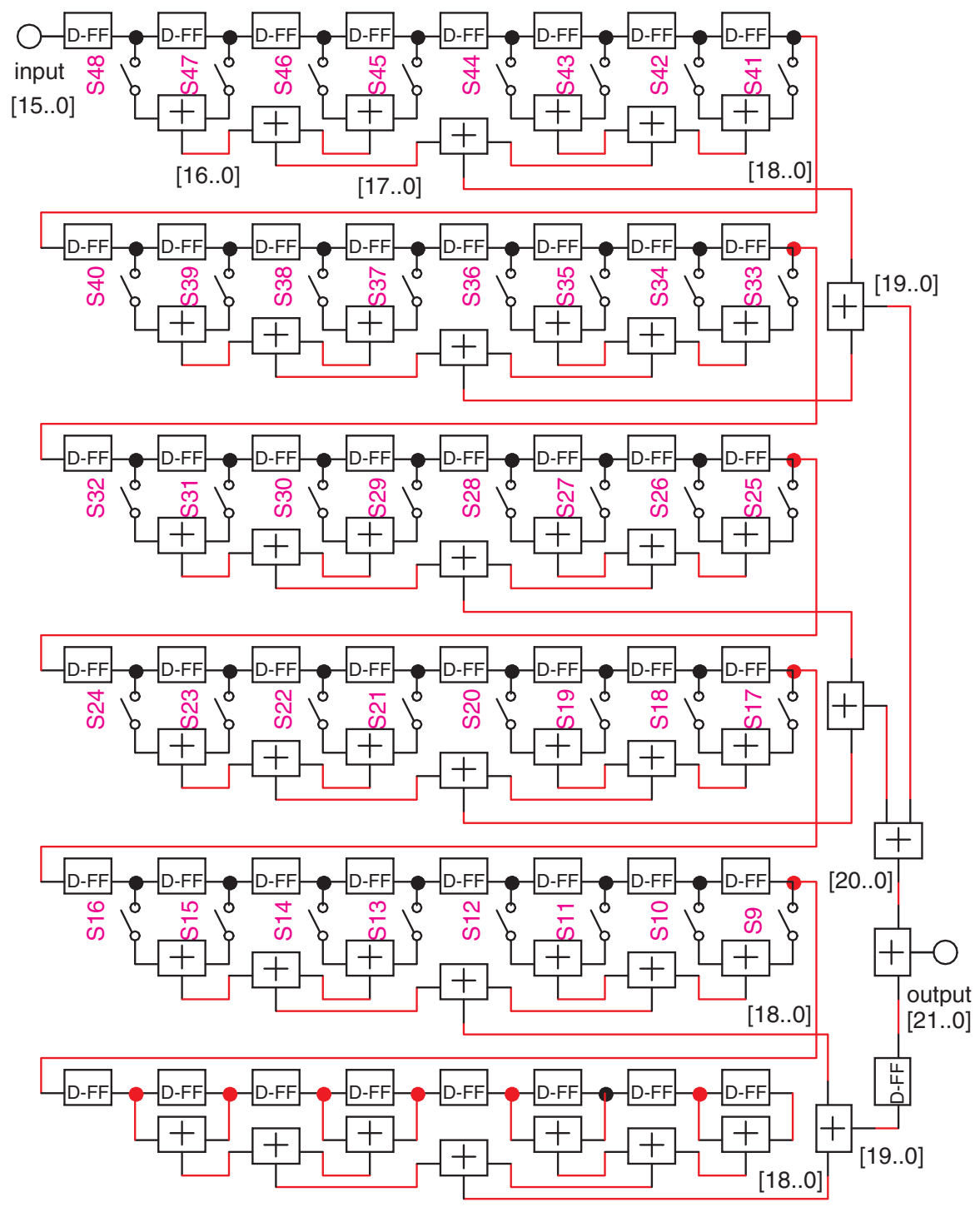

FIG. 15. (Color) The structure of the variable delay. For low frequency at RCS injection the switches S9 to S44 are closed. 


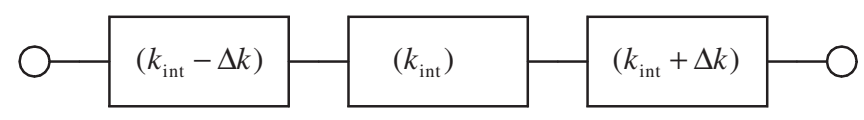

FIG. 16. Cascading 3 CIC filters with different $k_{\text {int }}$ in series.

\section{Cascading 3 interpolating CIC filters}

Using one of these interpolating CIC filters is not sufficient for good suppression of unwanted signals. For the digital receiver subsystem, which is operating with 16-bit arithmetic, we expect attenuation in the order of $80 \mathrm{~dB}$ or better. Also the notch width is not wide enough, using the maximum synchrotron frequency of RCS (approximately $6 \mathrm{kHz})$ as criterion. Increasing the number of filters put in series improves the notch depth. Selecting a different number of taps for each of the cascaded filters increases the notch width. As a good compromise between total delay and desired transfer function, in total 3 filters with different variable delay are put in series. According to Fig. 16 the original delay variable $\left(k_{\text {int }}\right)$ is replaced by either $\left(k_{\text {int }}-\Delta k\right)$ or $\left(k_{\text {int }}+\Delta k\right)$.
The value $\Delta k$ depends slightly on the revolution frequency as is shown in Fig. 17. Near RCS injection, at low frequency, the filter stop band is narrow. There $\Delta k=3$ is chosen. For revolution frequencies higher than $600 \mathrm{kHz}$, $\Delta k=2$ gives better performance.

This criterion can be expressed as function of $k_{\text {int }}$ :

For a value of $k_{\text {int }} \leq 60$, choose $\Delta k=2$.

For a value of $k_{\text {int }}>60$, choose $\Delta k=3$.

In case the injection energy of RCS is upgraded to $400 \mathrm{MeV}$, the value $\Delta k=2$ will stay constant during the whole RCS cycle.

\section{DISCUSSION OF RESULTS}

\section{A. Simulation of the 3 filters in series}

The filter structure, shown in Fig. 16 was simulated with SCILAB [18]. For the case $k_{\text {int }}=76$ and $\Delta k=3$ at injection, the filter length in total sample points is $(76-3+$

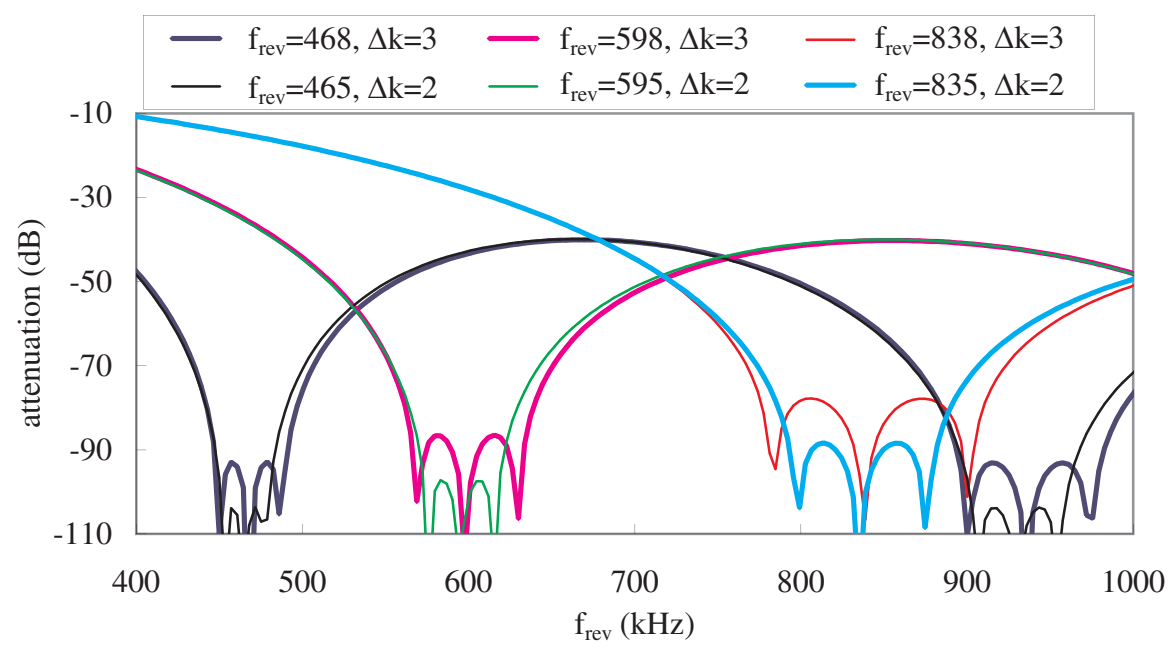

FIG. 17. (Color) The choice of $\Delta k$ influences the performance of the 3 variable CIC filters in series.

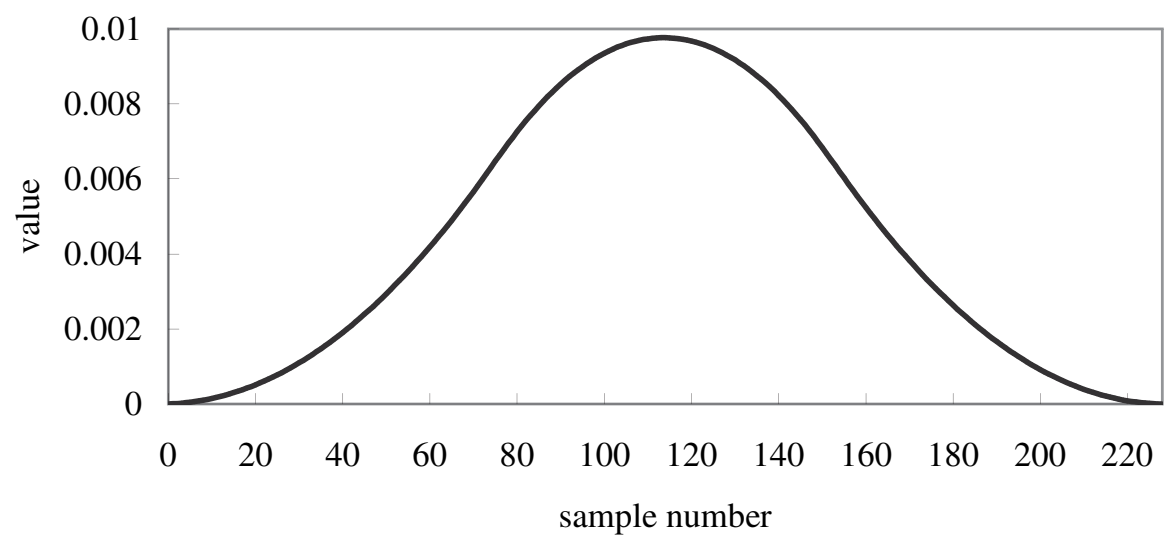

FIG. 18. The impulse response of 3 interpolating CIC filters in series for $k_{\text {int }}=76$ and $\Delta k=3$ has a length of 228 samples (equivalent to 228 clock cycles). 


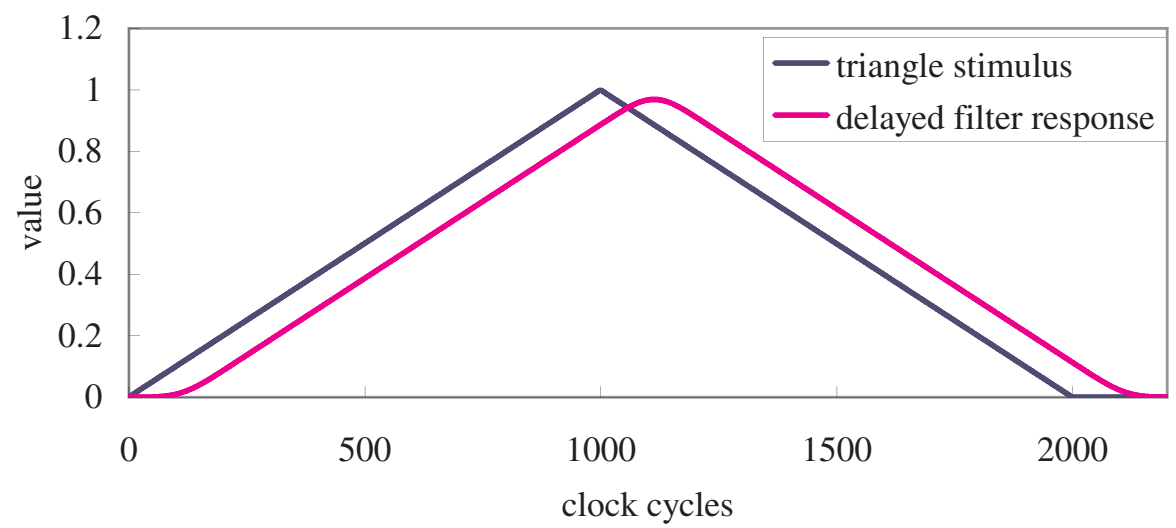

FIG. 19. (Color) The delay of 3 cascaded CIC filters with variable number of taps, arranged as $\left(k_{\text {int }}-3\right)\left(k_{\text {int }}\right)\left(k_{\text {int }}+3\right)$ at $469 \mathrm{kHz}$ for $k_{\text {int }}=76$ is 114 clock cycles $\Leftrightarrow 3.166 \mu \mathrm{s}$.

$76+76+3)=228$ taps. The impulse response is shown in Fig. 18. In the real circuit, the delay is slightly longer due to latches in the adder trees.

When a ramp with a rise time of 1000 clock cycles $=$ $27.75 \mu \mathrm{s}$ is applied as stimulus to the filter, the delay of the filter is half the number of total taps. At 50\% signal level, the answer of the filter is delayed by 114 clock cycles or $3.166 \mu$ s. This is shown in Fig. 19. For higher revolution frequency, the number of taps goes down, but the delay will not become shorter, because the delay flipflops are still in the signal path. This way, the behavior of the phase loop, where the variable CIC filter is used, does not change so much during the acceleration cycle. The resulting filter function at injection is shown in Fig. 20.

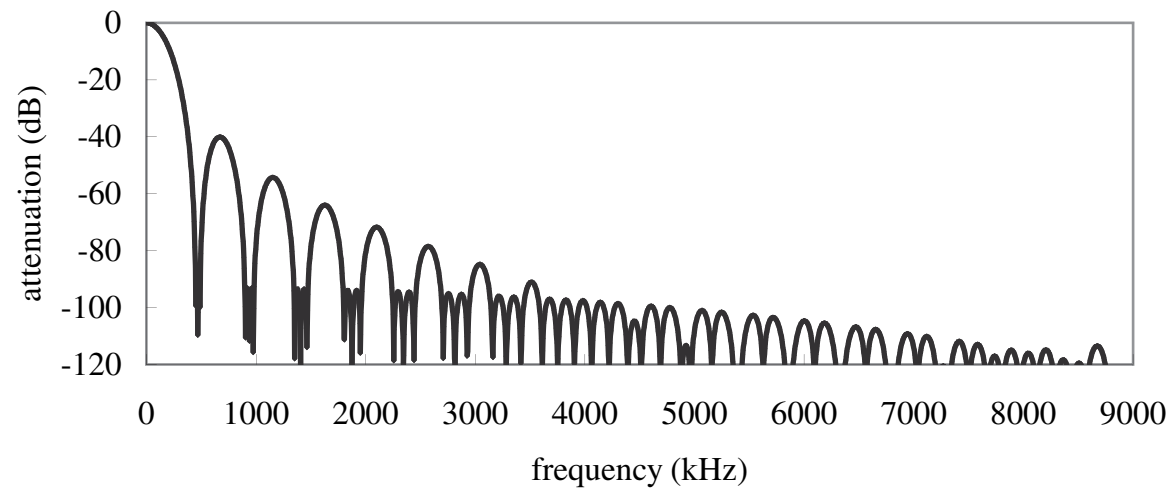

FIG. 20. CIC-filter function in frequency domain at injection for $\Delta k=3$.

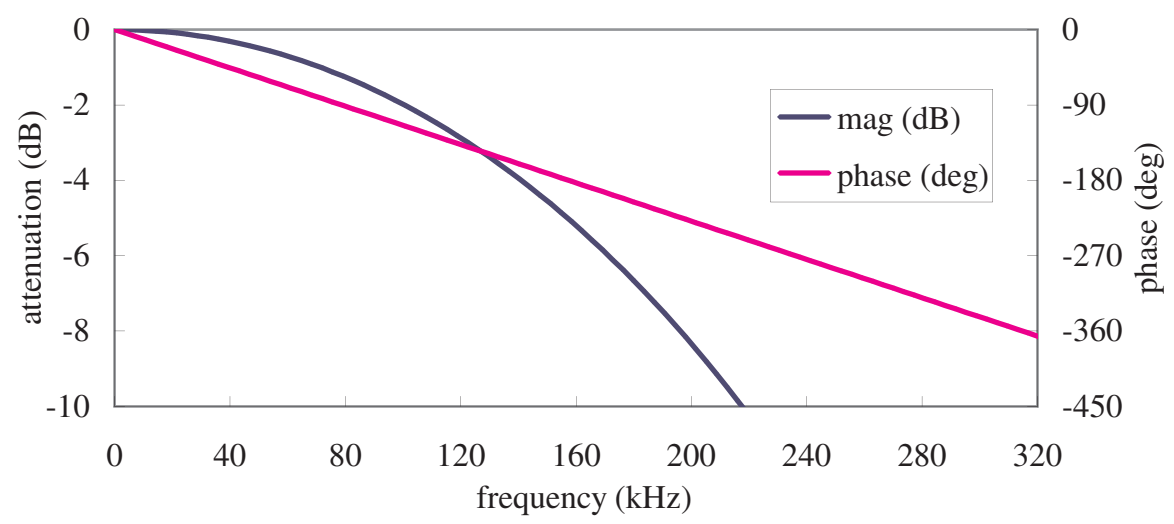

FIG. 21. (Color) Pass band of the combined CIC-filter function at $469 \mathrm{kHz}$ for $\Delta k=3$. The phase is $-90^{\circ}$ at $79 \mathrm{kHz},-140^{\circ}$ at $122.4 \mathrm{kHz}$ (3 dB point), and $-360^{\circ}$ at $315 \mathrm{kHz}$. 


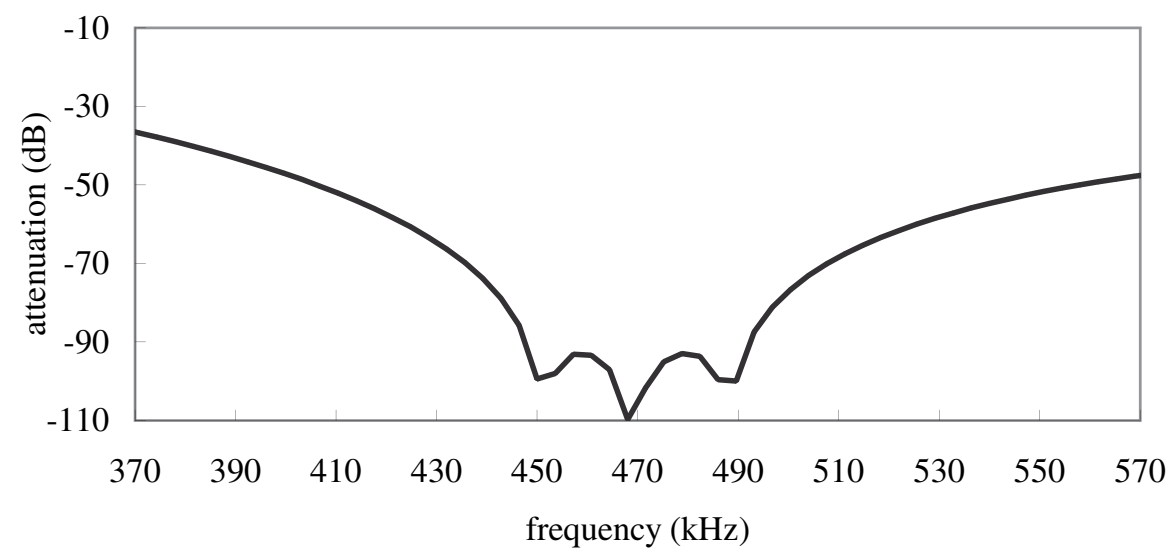

FIG. 22. Suppression of next harmonic of cascaded CIC filter for $\Delta k=3$ at injection.

Then Fig. 21 shows the pass band, and Fig. 22 the suppression of the next revolution harmonic, which is better than $90 \mathrm{~dB}$.

\section{B. Comparing with a standard FIR filter}

The digital low-pass filter for amplitude control in RCS [19] uses a combination of 3 CIC filters with 8 taps each (Fig. 23) for down sampling from $36 \mathrm{MHz}$ clock to $9 \mathrm{MHz}$ clock followed by a 63 tap FIR filter running at $9 \mathrm{MHz}$. The FIR filter has 2 delays between each coefficient, so that the length of the FIR filter is equivalent to $63 \cdot 8=504$ clock cycles at $36 \mathrm{MHz}$ clock. The FIR-filter coefficients are shown in Fig. 24. To allow for troubleshooting, the welltested FIR filter for amplitude control is also available for the phase-control circuit as a back-up solution. Both, the FIR and the variable CIC filter are realized in the same FPGA and are selected at boot time of the FPGA for phase control.

Figure 25 shows the response of the FIR-filter function in Fig. 24 to a ramp as stimulus. The same type of stimulus

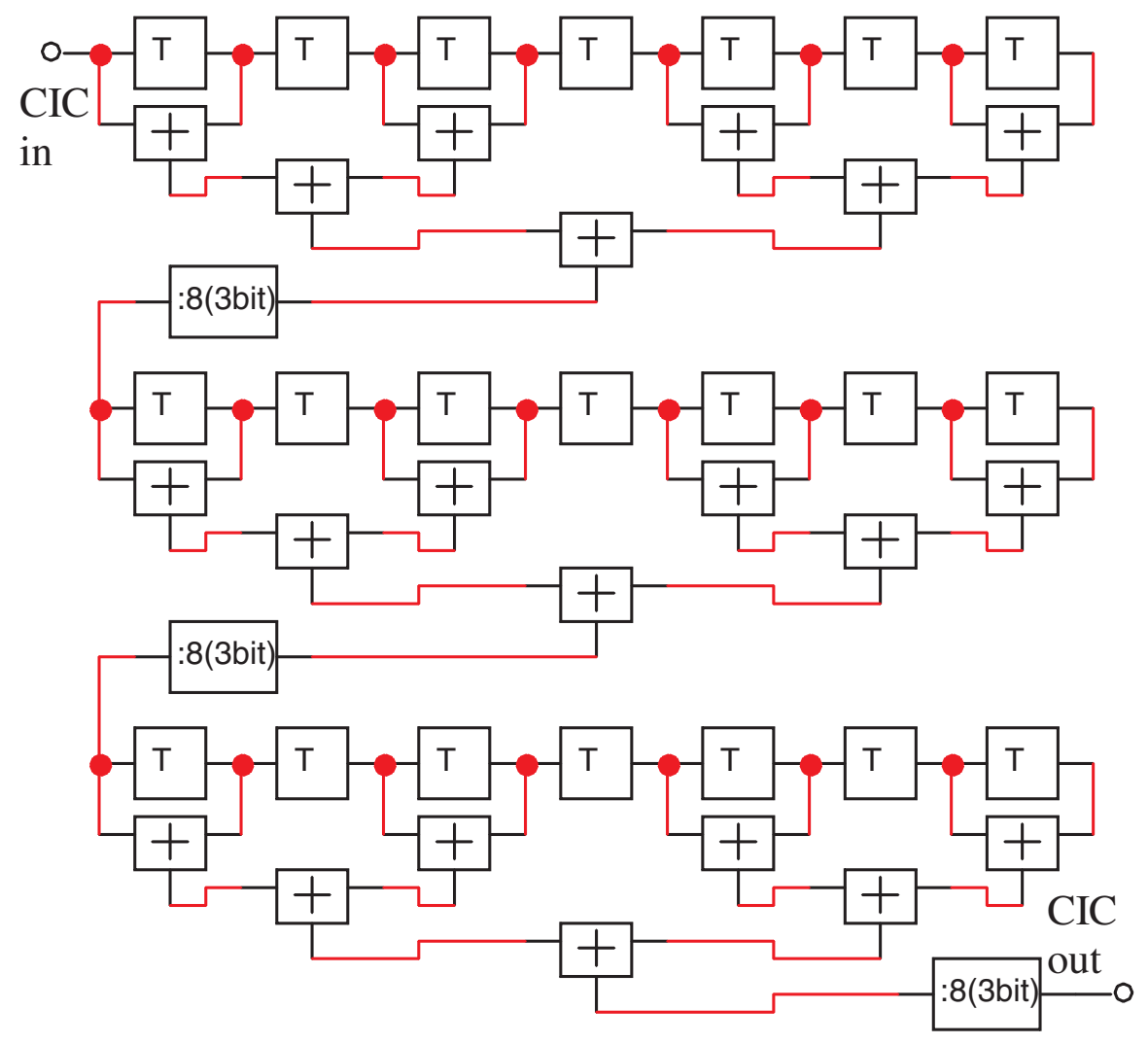

FIG. 23. (Color) Three CIC filters with 8 taps each for down sampling from 36 to $9 \mathrm{MHz}$. 


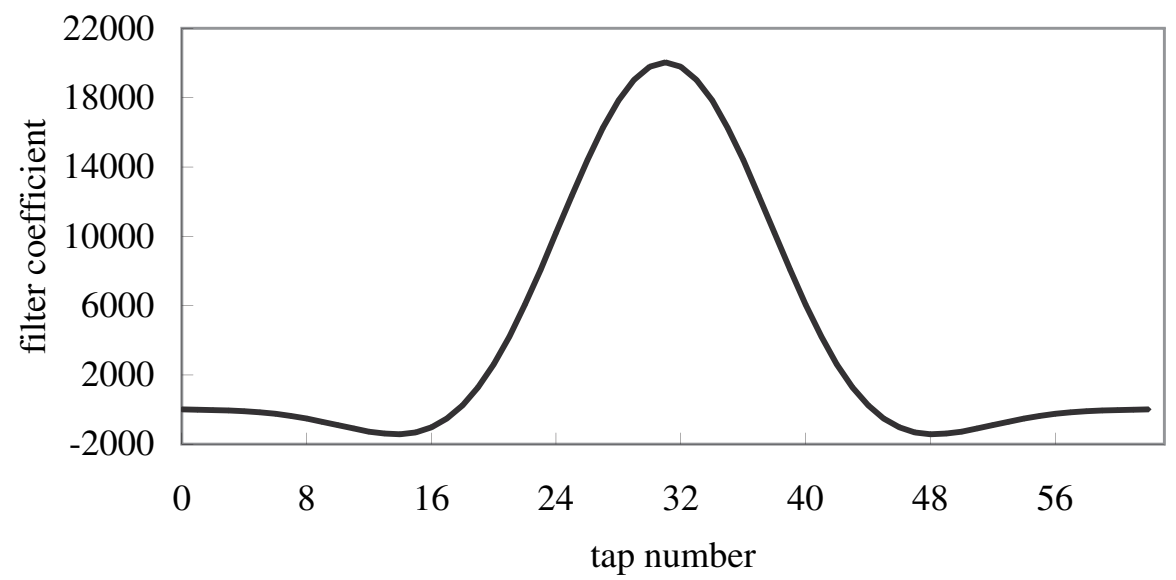

FIG. 24. FIR-filter coefficients for RCS amplitude control: 63 symmetric taps at $9 \mathrm{MHz}$ clock with 2 delays between each tap (equivalent to 504 cycles at $36 \mathrm{MHz}$ clock).

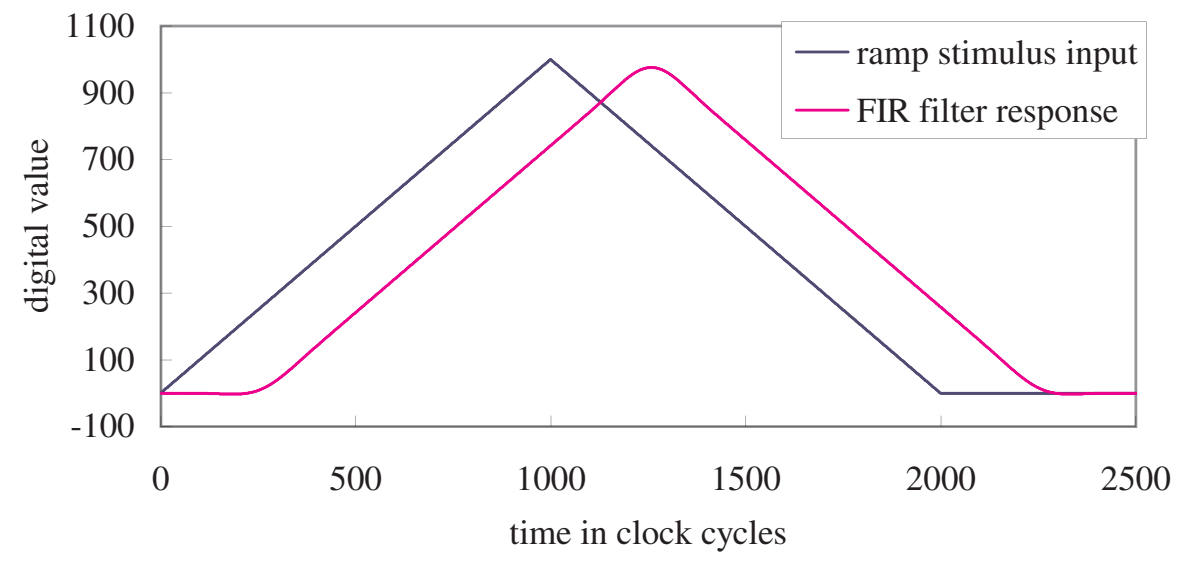

FIG. 25. (Color) Delay of the FIR filter with effectively 504 taps at $36 \mathrm{MHz}$ clock. Stimulus is a 1000-point ramp (up/down) at $36 \mathrm{MHz}$ clock with $27.77 \mu$ s rise time. At half pulse height the delay is 258.5 clocks $=7.18 \mu \mathrm{s}$.

as in Fig. 19 for the variable CIC filter is applied. The FIRfilter delay is more than twice compared to the variable CIC filter in Fig. 19. The frequency characteristics of the FIR filter combined with the CIC filter for down sampling are shown in Fig. 26(a) from dc to the Nyquist frequency limit. The FIR filter is clocked with $9 \mathrm{MHz}$, therefore Fig. 26(b) confirms, that the CIC down-sampling filter provides enough attenuation at multiples of $4.5 \mathrm{MHz}$. Then Fig. 26(c) shows the pass-band characteristic. The FIR filter has a longer delay, compared to the variable CIC filter, because the total frequency span that is attenuated is wider.

\section{Simulation results with a phase loop}

The digital phase loop for RCS is simulated with PSPICE to check the stability margin [20]. For $6 \mathrm{kHz}$ synchrotron frequency, both control loops, either with FIR low-pass filter for down conversion [Fig. 27(a)], or with variable CIC filter [Fig. 28(a)] are stable. For an assumed synchrotron frequency of $30 \mathrm{kHz}$, the loop with the FIR filter
[Fig. 27(b)] is near instability and shows strong ringing, while the phase loop with the variable CIC filter [Fig. 28(b)] is stable. The reason for the smaller stability margin of the FIR filter is the longer delay, which translates into a phase shift of $-90^{\circ}$ at $33.89 \mathrm{kHz}$.

\section{SAVING FPGA RESOURCES BY DOWN SAMPLING}

One CIC filter will need $82 \times 16$-bit D-FF for the delays, 4 multipliers in the delay circuit, and 79 adders in the adder tree. For 3 of the variable CIC filters in series, running at $36 \mathrm{MHz}$ clock, approximately $250 \times 16$-bit DFF, 12 multipliers, 250 adders, and the table to look up for 40 scale values are needed. There are several possibilities to save resources.

\section{A. Optimizing the circuit}

The 3 filters in series in Fig. 16 have different numbers of taps, for example, $\left(k_{\mathrm{int}}-2\right),\left(k_{\mathrm{int}}\right)$, and $\left(k_{\mathrm{int}}+2\right)$. The 

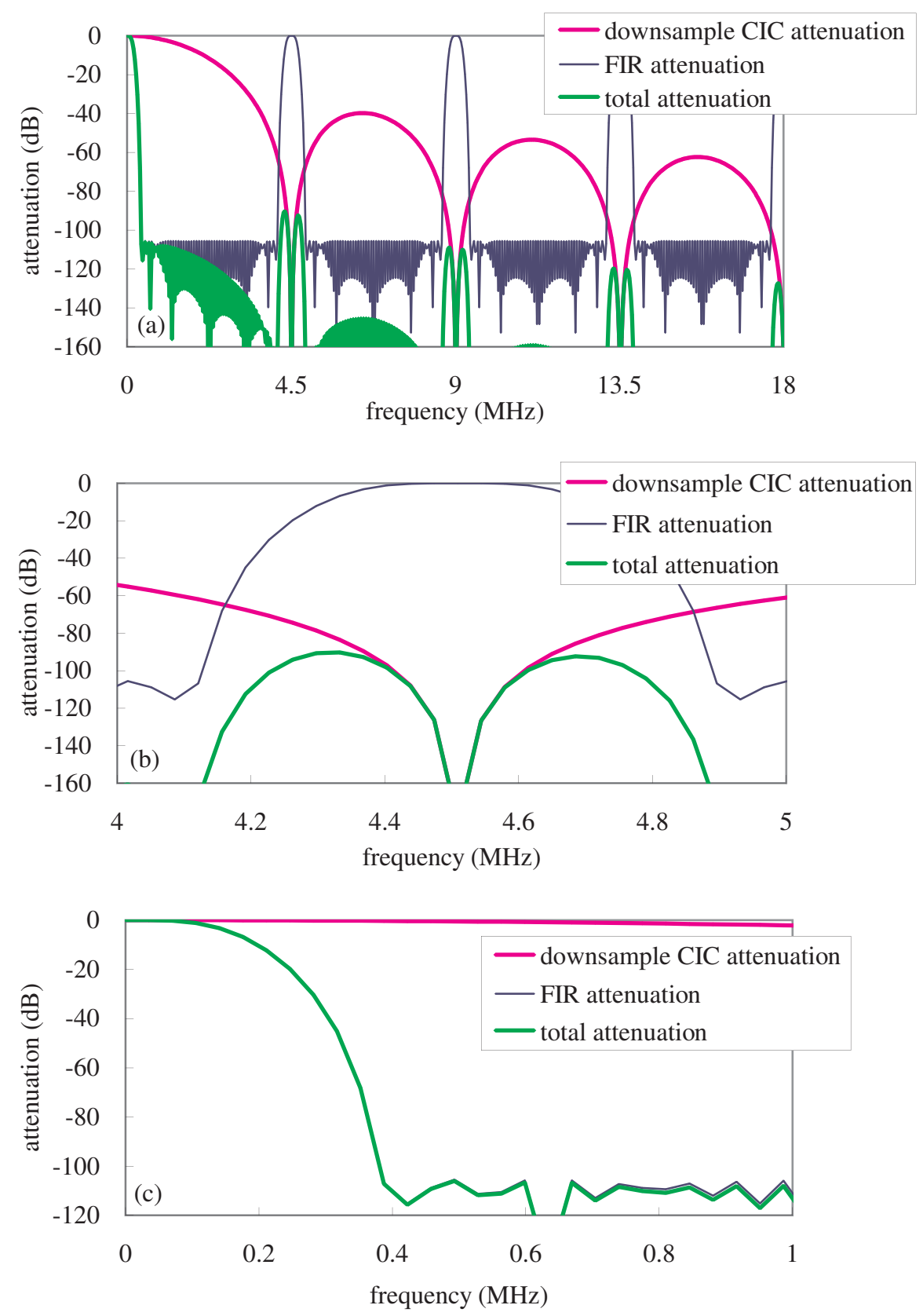

FIG. 26. (Color) (a) Transfer function of the low-pass filter for amplitude control, consisting of a $3 \times 8$ tap CIC for down sampling, and a FIR with 2 delays at each of the 63 taps at $9 \mathrm{MHz}$. (b) Attenuation at Nyquist rate of the FIR filter clocked at 9 MHz. Enlarged view of (a) around 4.5 MHz. (c) Pass-band characteristic of the Low-pass filter for amplitude control. Enlarged view of (a) from de to $1 \mathrm{MHz}$.

maximum value for $\left(k_{\text {int }}\right)$ is 76 at injection. It is possible to remove always unused taps in the filters with $\left(k_{\text {int }}\right)$ and $\left(k_{\text {int }}-2\right)$. This way, $6 \ldots 8 \times 16$-bit D-FF can be saved. Also the total delay will be approximately $200 \mathrm{~ns}$ shorter. As a disadvantage, each CIC-filter stage will look different.

\section{B. Down sampling to $9 \mathrm{MHz}$ clock frequency}

This approach is the same as reducing the resources for the FIR filter for amplitude control, shown in Fig. 24.
The 3-stage CIC filter with 8 taps from Fig. 23 at the input sides provides low-pass filtering, so that the sample rate can be reduced to $9 \mathrm{MHz}$. However, for the 3 variable CIC filters in series, $\Delta k$ has to be smaller than 1 . In case of $\Delta k=1$ the spacing between the harmonics for $\left(k_{\text {int }}\right)$ and $\left(k_{\text {int }} \pm 1\right)$ becomes too big. Therefore the difference in the number of taps between the filter stages $\Delta k$ becomes noninteger. An example with $\left(k_{\text {int }} \pm 0.5\right)$ is shown in Fig. 29. Some translation logic for the filter with $\left(k_{\text {int }} \pm 0.5\right)$ is necessary, which makes the circuit 

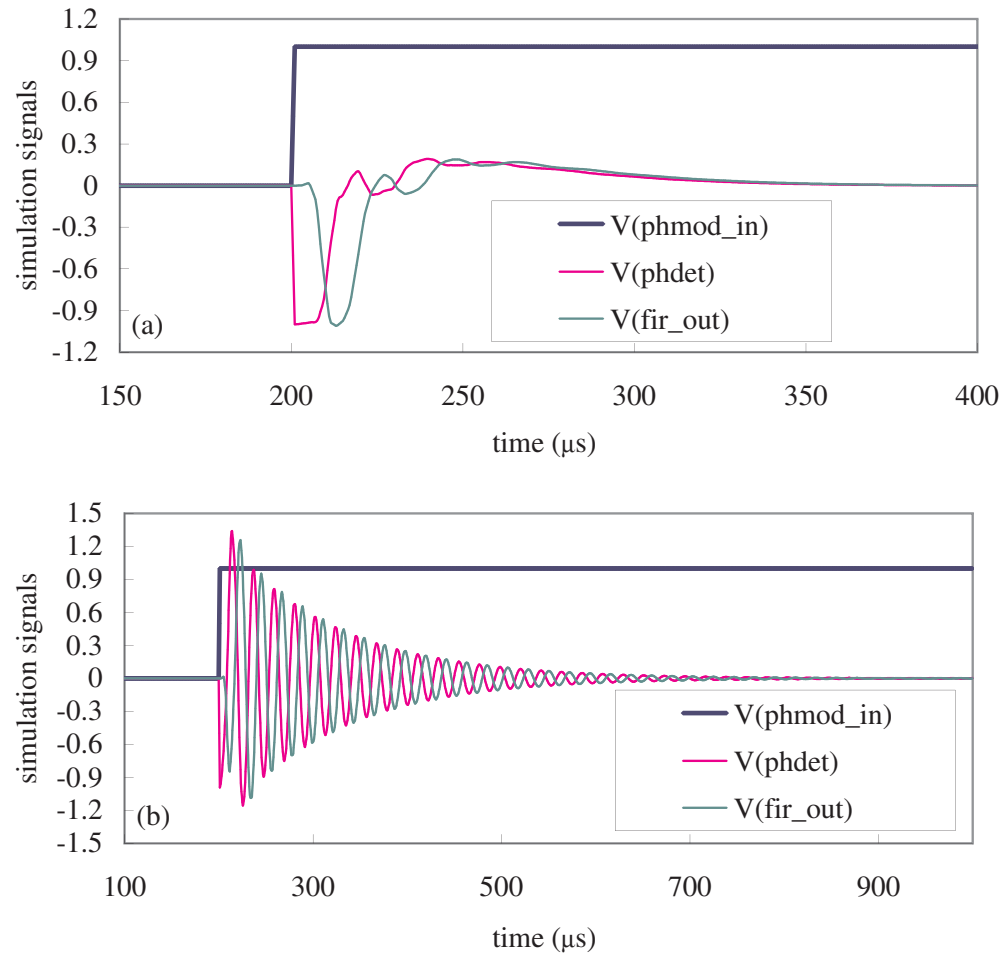

FIG. 27. (Color) (a) Simulated phase loop with FIR low-pass filter is stable at $6 \mathrm{kHz}$ synchrotron frequency. (b) Simulated phase loop with FIR low-pass filter is near instability and shows ringing at $30 \mathrm{kHz}$ synchrotron frequency. In both figures, the stimulus is a phasejump with $1 \mu$ s rise time.
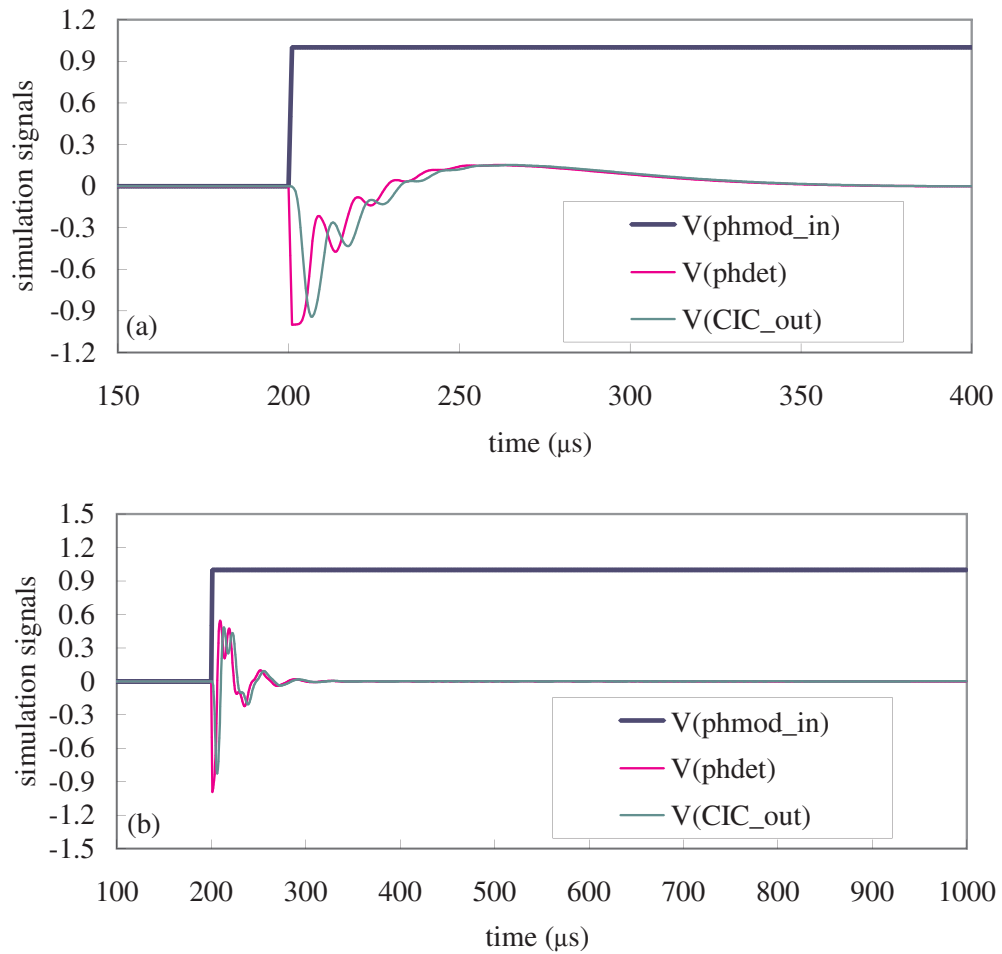

FIG. 28. (Color) (a) Simulated phase loop with variable CIC low-pass filter is stable at $6 \mathrm{kHz}$ synchrotron frequency. (b) Simulated phase loop with variable CIC low-pass filter is stable at $30 \mathrm{kHz}$ synchrotron frequency. In both figures, the stimulus is a phase-jump with $1 \mu$ s rise time. 


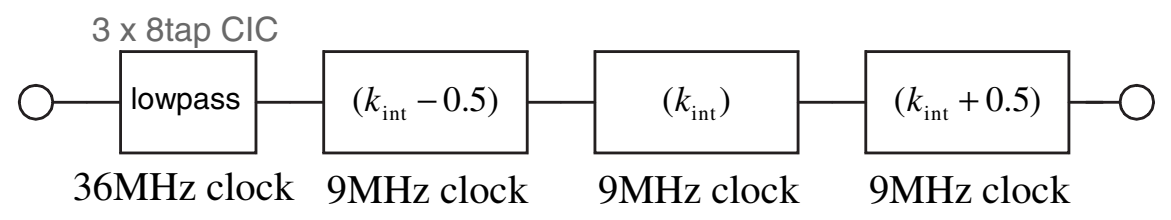

FIG. 29. Down sampling to $9 \mathrm{MHz}$ to reduce resources results in noninteger $\Delta k$.

more difficult to understand, and less efficient in terms of used resources.

\section{Down sampling to $18 \mathrm{MHz}$ clock frequency}

As in Sec. VB and Fig. 23, a 3-stage CIC filter with 8 taps is used as a prefilter. For the phase-feedback board [21] (PFB) it reduces the sample rate from 36 to $18 \mathrm{MHz}$ (see Fig. 30). This prefilter introduces an additional delay of 36 clock cycles. 12 of these clock cycles are delays in the adder tree, and 24 of these delays are related to the filter taps. For slow signals like a ramp, the delays in the filter taps only contribute half. Then the 3 -stage 8 tap CIC filter introduces a delay of $12+24 / 2=24$ clocks, or $666 \mathrm{~ns}$ for ramp signals as stimulus. This filter already exists in the FPGA for the FIR filter (Sec. IV B), which is the back-up solution-therefore its design is reused. One CIC filter will need approximately: $55 \mathrm{D}-\mathrm{FF}$ for the delays, 4 multipliers, and 41 adders for the delays. For the 3 CIC filters in series, running at $18 \mathrm{MHz}$ clock, approximately $165 \times$ 16-bit D-FF, 12 multipliers, and 123 adders, and the table to look up for 32 scale values are needed. The CIC-stages for down sampling need another $24 \mathrm{D}-\mathrm{FF}$ for delay, and 21 adders.
In discussions with the company, who is building the RCS digital low-level rf system, the structure in Fig. 30 was defined as the preferred filter for phase control in RCS. In the $36 \mathrm{MHz}$ version, the variable $\Delta k$ was either 2 or 3 , depending on revolution frequency. In the $18 \mathrm{MHz}$ version, $\Delta k$ is constant with $\Delta k=1$. The circuit for the single variable CIC filter then changes from Fig. $12(36 \mathrm{MHz}$ clock version) to Fig. 31 (18 MHz clock version).

With the reduced number of delays, the look-up table for $k_{\text {int }}\left(f_{\text {rev }}\right)$ (Table I) is modified to Table V. Also the scaling factor (Table III) is changed according to Table VI. The 18bit value for $k_{\text {int }}=16$ is reduced by 1 , so that it fits within 18-bit. Table VII shows the limits of $\left(k_{\text {int }}+\Delta k\right)$ for the 6 places where these values appear.

The transfer function of the 3 interpolated CIC filters (18 $\mathrm{MHz}$ version) is shown for 3 different revolution frequencies in Fig. 32. Figure 33(a) shows the attenuation of the combination of CIC prefilter and 3 variable CIC filters (the structure of Fig. 30) from dc up to $18 \mathrm{MHz}$ - the Nyquist frequency for a $36 \mathrm{MHz}$ clock system. Figure 33(b) gives an enlarged view of the frequency span from dc to $4.5 \mathrm{MHz}$. Thanks to the prefiltering, the suppression of the higher harmonics of the revolution frequency is $90 \mathrm{~dB}$ or better, which is an improvement

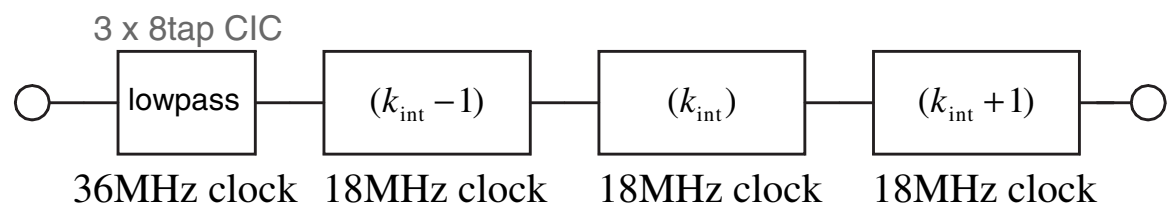

FIG. 30. Down sampling to $18 \mathrm{MHz}$ clock reduces used resources. $\Delta k=1$ is kept constant during the RCS frequency sweep.

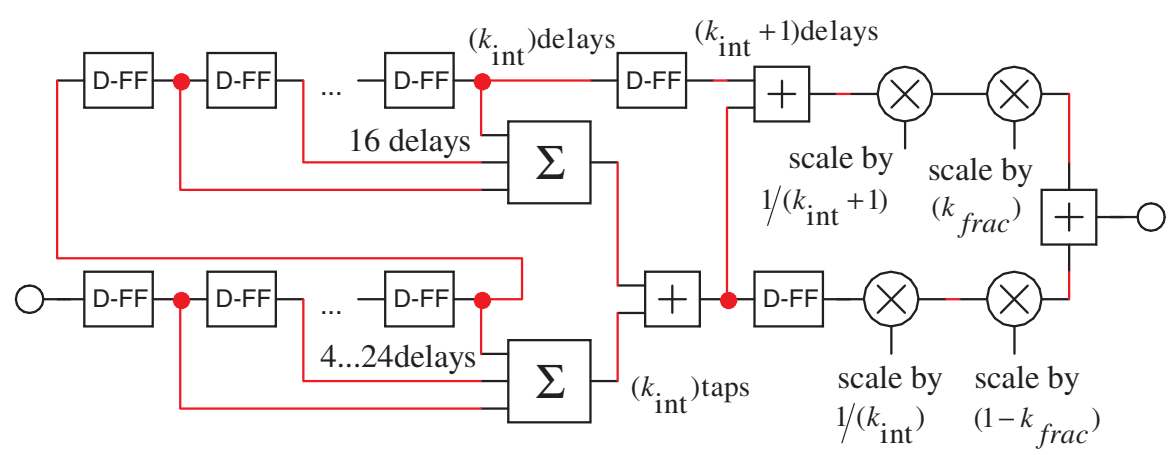

FIG. 31. (Color) The interpolated CIC filter for variable relation $k_{\mathrm{opt}}$ between $18 \mathrm{MHz}$ clock frequency and revolution frequency. 
TABLE V. Examples of the filter pattern for selected frequencies $f_{\text {rev }}$ for the $18 \mathrm{MHz}$ filter version.

\begin{tabular}{lllllllll}
\hline \hline$f_{\text {rev }}(\mathrm{kHz})$ & \multicolumn{1}{c}{$k_{\text {opt }}$} & $k_{\text {int }}$ & $k_{\text {int }}-16$ & $k_{\text {int }}-16(\mathrm{MSB})$ & $k_{\text {frac }}$ & Int $\left(1024 k_{\text {frac }}\right)$ & Remain (LSB) & Pattern (hex) \\
\hline 439.025 & 40.999943 & 40 & 24 & 6000 & 0.999943 & 1023 & $03 \mathrm{FF}$ & $63 \mathrm{FF}$ \\
450.000 & 40 & 40 & 24 & 6000 & 0.0 & 0 & 0000 & 6000 \\
$\mathbf{4 6 9 . 2 5 0}$ & 38.359084 & 38 & 22 & 5800 & 0.359084 & 367 & $016 \mathrm{~F}$ & $596 \mathrm{~F}$ \\
500.000 & 36 & 36 & 20 & 5000 & 0.0 & 0 & 0000 & 5000 \\
$\mathbf{6 1 3 . 6 9 1}$ & 29.33072 & 29 & 13 & 3400 & 0.33072 & 338 & 0152 \\
750.000 & 24 & 24 & 8 & 2000 & 0.0 & 0 & 0000 & 2000 \\
\hline \hline
\end{tabular}

TABLE VI. Selected values of the table for the scaling factor $\left(1 / k_{\text {int }}\right)$ for the $18 \mathrm{MHz}$ filter version.

\begin{tabular}{lcccc}
\hline \hline$k_{\text {int }}-16$ & $k_{\text {int }}$ & $2^{21} / k_{\text {int }}$ & 18-bit signed & Hex \\
\hline 0 & 16 & 131072.0 & 131071 & 1 FFFF \\
1 & 17 & 123361.88 & 123361 & $1 \mathrm{E} 1 \mathrm{E} 1$ \\
4 & 20 & 104857.6 & 104857 & 19999 \\
24 & 40 & 52428.8 & 52428 & CCCC \\
31 & 47 & 44620.255 & 44620 & AE4C \\
\hline \hline
\end{tabular}

compared to the $36 \mathrm{MHz}$ version without down sampling in Fig. 17. Of course, an attenuation of $100 \mathrm{~dB}$ or more as shown in Figs. 32 and 33 is only possible with floatingpoint arithmetic. With $16 \ldots 18$-bit integer arithmetic used in the FPGA, the attenuation is limited to $90 \ldots 100 \mathrm{~dB}$.

\section{APPLICATION: 90 ${ }^{\circ}$ DELAY (HILBERT- TRANSFORMATION)}

The basic idea for the interpolating CIC filter can be applied for solving the problem of generating a delay, that is proportional to the revolution period of a particle beam. This allows "one-turn-delays" [22] or $90^{\circ}$ delays for Hilbert transformation to be realized with a fixed clock digital system.

Figure 34 shows the structure of a digital $90^{\circ}$ phase shifter for Hilbert transformation that can follow the revolution frequency while operating at fixed clock. The optimum number of delays for $90^{\circ}$ at harmonic $h$ is

$$
\left(k_{\mathrm{opt}}\right)=\frac{f_{\mathrm{clk}}}{4 \cdot h \cdot f_{\mathrm{rev}}} .
$$

The integer number of delays is $\left(k_{\text {int }}\right)=\operatorname{int}\left(k_{\text {opt }}\right)$. The corresponding scaling factors $a_{1}$ and $a_{2}$ are computed in

TABLE VII. Minimum and maximum for $\left(k_{\text {int }}+\Delta k\right)$ while accessing Table VI.

\begin{tabular}{lcccccr}
\hline \hline & \multicolumn{2}{c}{ Filter with $\Delta k=-1$} & \multicolumn{2}{c}{ Filter with $\Delta k=0$} & \multicolumn{2}{c}{ Filter with $\Delta k=1$} \\
& $k_{\text {int }}+\Delta k$ & $k_{\text {int }}+\Delta k+1$ & $k_{\text {int }}$ & $k_{\text {int }}+1$ & $k_{\text {int }}+\Delta k$ & 22 \\
Minimum & 20 & 21 & 21 & 22 & 39 & 23 \\
Maximum & 37 & 38 & 38 & 39 & 40 \\
\hline \hline
\end{tabular}

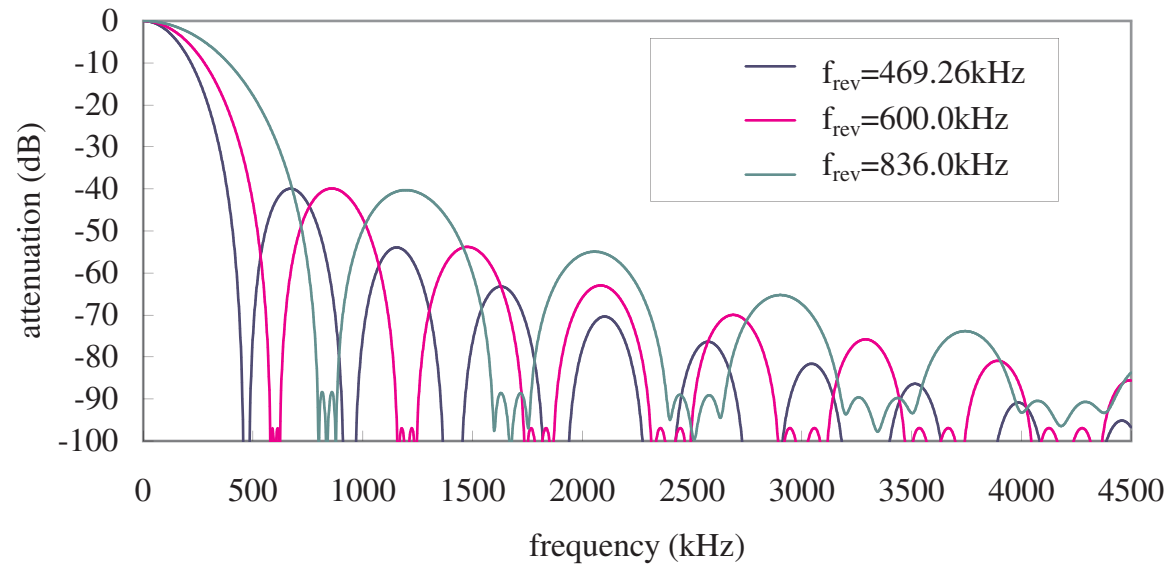

FIG. 32. (Color) Attenuation of 3 combined CIC filters with $\Delta k=(-1 ; 0 ;+1)$ at $18 \mathrm{MHz}$ sample rate. The effect of the downsampling $\mathrm{CIC}$ at the input is not included. 

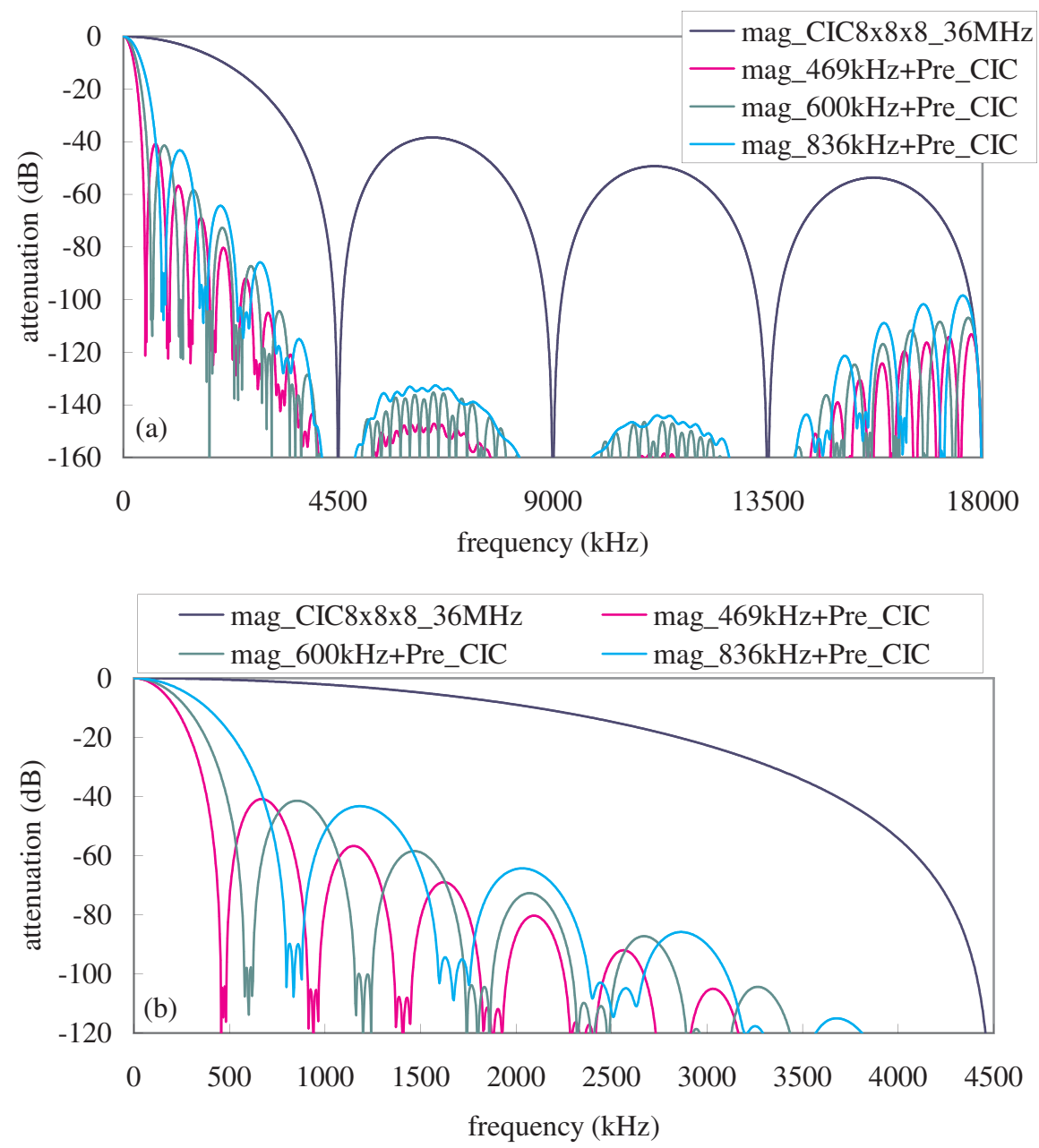

FIG. 33. (Color) (a) Attenuation of down-sampling CIC (to $18 \mathrm{MHz}$ ) combined with 3 variable CIC filters at $18 \mathrm{MHz}$ sample rate. (b) The attenuation of the higher harmonics is shown in a reduced frequency span from dc to $4.5 \mathrm{MHz}$ of (a).

a way, that the amplitude at the output follows the input amplitude and the phase between input and output is $90^{\circ}$ as expected.

The output after $\left(k_{\text {int }}\right)$ delays is a complex vector $z_{1}=$ $x_{1}+j y_{1}$, and the output after $\left(k_{\text {int }}+1\right)$ delays is a complex vector $z_{2}=x_{2}+j y_{2}$. After scaling with the real numbers $a_{1}$ and $a_{2}$, we get the sum in frequency domain

$$
S(f)=a_{1} \cdot z_{1}+a_{2} \cdot z_{2}=a_{1}\left(x_{1}+j y_{1}\right)+a_{2}\left(x_{2}+j y_{2}\right) .
$$

For a $90^{\circ}$ phase shift, the real part has to be zero

$$
a_{1} \cdot x_{1}+a_{2} \cdot x_{2}=0,
$$

and the amplitude has to be constant, e.g., 1 .

$$
\left(a_{1} x_{1}+a_{2} x_{2}\right)^{2}+\left(a_{1} y_{1}+a_{2} y_{2}\right)^{2}=1 .
$$

Equation (11) inserted into (12) simplifies to

$$
a_{1} y_{1}+a_{2} y_{2}= \pm 1
$$

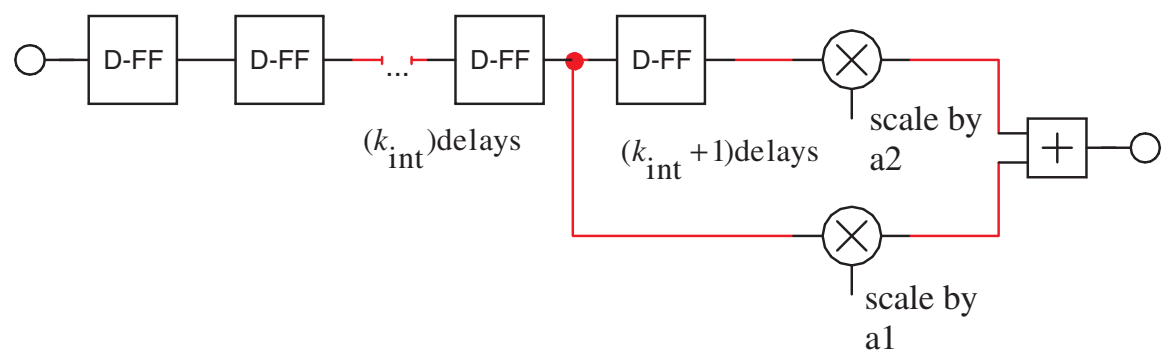

FIG. 34. (Color) Structure of a digital $90^{\circ}$ phase shifter for Hilbert transformation. 


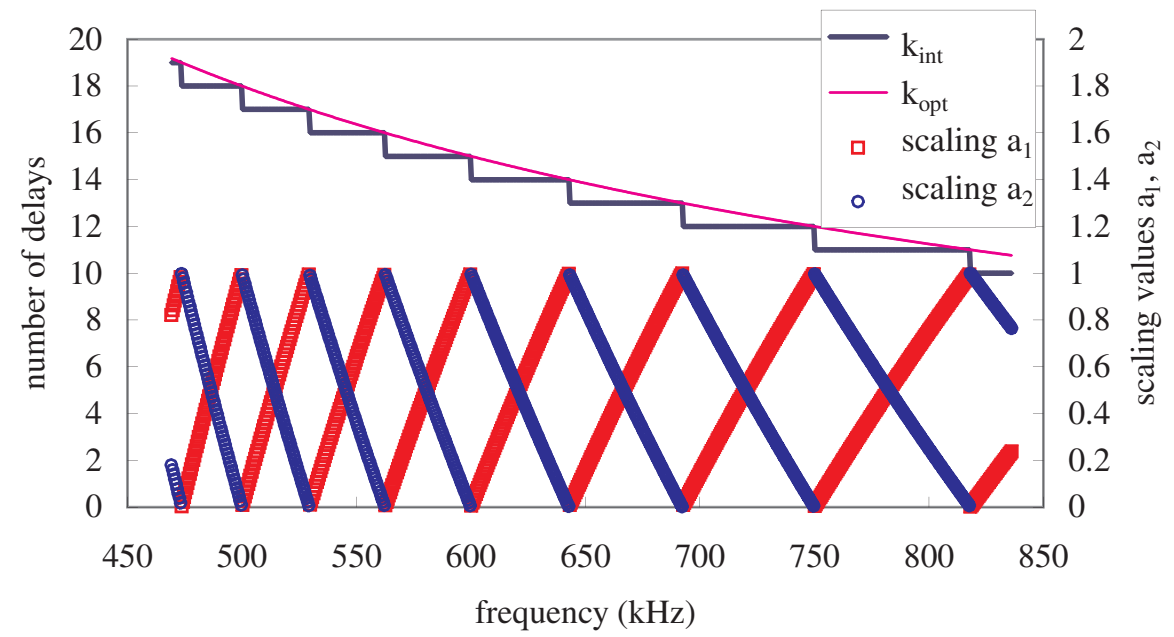

FIG. 35. (Color) Scaling factors to be stored in a pattern memory for a Hilbert transformer tracking the RCS cycle at $(h=1)$.

$$
a_{1}=\frac{\mp x_{2}}{y_{2} x_{1}-y_{1} x_{2}} ; \quad a_{2}=\frac{ \pm x_{1}}{y_{2} x_{1}-y_{1} x_{2}} .
$$

Now we insert the transfer function of the delays

$$
\begin{aligned}
z_{1}= & e^{-j 2 \pi h \cdot k_{\mathrm{int}} \cdot f / f_{\mathrm{clk}}} \\
= & \cos \left(-2 \pi h \cdot k_{\mathrm{int}} \cdot f / f_{\mathrm{clk}}\right) \\
& +j \sin \left(-2 \pi h \cdot k_{\mathrm{int}} \cdot f / f_{\mathrm{clk}}\right), \\
z_{2}= & e^{-j 2 \pi h \cdot\left(k_{\mathrm{int}}+1\right) \cdot f / f_{\mathrm{clk}}} \\
= & \cos \left(-2 \pi h \cdot\left(k_{\mathrm{int}}+1\right) \cdot f / f_{\mathrm{clk}}\right) \\
& +j \sin \left(-2 \pi h \cdot\left(k_{\mathrm{int}}+1\right) \cdot f / f_{\mathrm{clk}}\right) .
\end{aligned}
$$

The variation of $\left(k_{\text {int }}\right)$ and the amplitude scaling factors $a_{1}$ and $a_{2}$ for the frequency swing of RCS at $(h=1)$ is shown in Fig. 35. $z_{1}=x_{1}+j y_{1}$, and $z_{2}=x_{2}+j y_{2}$ from Eqs. (15) and (16) were inserted into Eq. (14), and the upper solution was used to obtain positive scaling factors $a_{1}$ and $a_{2}$. The scaling factors $a_{1}$ and $a_{2}$, and the variable delay selector $\left(k_{\text {int }}\right)$ are put into a pattern memory, so that they can be accessed as function of revolution frequencyas in the case of the interpolated CIC filter.

\section{OUTLOOK}

The filter structure explained in this document provides a way to translate the idea of a CIC filter for variable clock into designs for fixed clock. In this way, a recipe for notch filters which track a reference frequency is given. These filters have a shorter delay compared to standard FIR applications, so that the stability of loops using these filters is improved. This is important for the high intensity proton synchrotron RCS, where beam loss has to be kept as small as possible. Both filter types (FIR and variable CIC) are realized in FPGA technology, so that we are able to test both configurations in the commissioning phase of RCS. We plan to use this filter structure for the main-ring (MR) of J-Parc, too. In addition, this filter design method can be applied to generate a $90^{\circ}$ delay for Hilbert transformation or a "one-turn delay" for fixed clock digital systems.

[1] Y. Irie, in Proceedings of the European Particle Accelerator Conference, Lucerne, 2004 (EPS-AG, Lucerne, 2004), p. 113.

[2] M. Yoshii, in Proceedings of the Particle Accelerator Conference, Knoxville, TN, 2005 (IEEE, Piscataway, NJ, 2005), pp. 475-477, http://accelconf.web.cern.ch/ AccelConf/p05/PAPERS/ROAC005.PDF.

[3] M. Yamamoto, M. Nomura, A. Schnase, F. Tamura, S. Anami, E. Ezura, K. Hara, Y. Hashimoto, C. Ohmori, A. Takagi, and M. Yoshii, in Proceedings of the Particle Accelerator Conference, Knoxville, TN, 2005 (Ref. [2]),pp. 931-933, http://accelconf.web.cern.ch/ AccelConf/p05/PAPERS/TPPT005.PDF.

[4] F. Tamura, M. Yamamoto, M. Nomura, A. Schnase, S. Anami, E. Ezura, K. Hara, C. Ohmori, A. Takagi, and M. Yoshii, in Proceedings of the Particle Accelerator Conference, Knoxville, TN, 2005 (Ref. [2]), pp. 36243626, http://accelconf.web.cern.ch/AccelConf/p05/ PAPERS/WPAT064.PDF.

[5] A. Schnase, M. Nomura, F. Tamura, M. Yamamoto, S. Anami, E. Ezura, K. Hara, C. Ohmori, A. Takagi, and M. Yoshii, in Proceedings of the Particle Accelerator Conference, Knoxville, TN, 2005 (Ref. [2]), pp. 10631065, http://accelconf.web.cern.ch/AccelConf/p05/ PAPERS/WPAT007.PDF.

[6] Alan V. Oppenheim and Ronald W. Schafer, Discrete-Time Signal Processing (Prentice-Hall, Englewood Cliffs, NJ, 1989), 1st ed., Vol. 1, Chap. 7.4-7.8, pp. 444-489.

[7] E. B. Hogenauer, in IEEE Trans. Acoust. Speech Signal Process. 29, 155 (1981).

[8] D. Boussard and G. Lambert, in Proceedings of the 1983 Particle Accelerator Conference, Santa Fe, New Mexico (IEEE, Piscataway, NJ, 1983), p. 2239. 
[9] D. Boussard, in Proceedings of the 1985 IEEE Particle Accelerator Conference, Vancouver, Canada (IEEE, Piscataway, NJ, 1985), p. 1852.

[10] P. McIntosh, M. Browne, J. Dusatko, J. Fox, W. Ross, D. Teytelman, and D. Van Winkle, in Proceedings of European Particle Accelerator Conference, Lucerne, Switzerland, 2004 (EPS-AG, Lucerne, 2004), p. 1087.

[11] S. Yoshimoto, E. Ezura, K. Akai, and T. Takashima, in Proceedings of the Particle Accelerator Conference, Dallas, TX, 1995 (IEEE, Piscataway, NJ, 1995), p. 2675.

[12] S. Yoshimoto, E. Ezura, and K. Akai, in Proceedings of the European Particle Accelerator Conference, Barcelona, Spain, 1996 (CRC Press, Boca Raton, FL, 1996), p. 1899.

[13] I. Janiszewski, H. Meuth, and B. Hoppe, in Proceedings of SOCC2004, Santa Clara, CA, 2004 (IEEE, Piscataway, NJ, 2004), pp. 373-376.

[14] R. Garoby, in CERN Accelerator School: RF Engineering for Particle Accelerators, edited by S. Turner (CERN, Geneva, 1992), Vol. 2, p. $428 \mathrm{ff}$.

[15] A. Schnase, Doctorate thesis, RWTH Aachen, Institut f. Hochfrequenztechnik, 1994.
[16] F. Tamura, J. Chiba, T. Katoh, and M. Yoshii, in Proceedings of the Particle Accelerator Conference, Knoxville, TN, 2005 (Ref. [2]), pp. 3853-3855, http:// accelconf.web.cern.ch/AccelConf/p05/PAPERS/ FPAT071.PDF.

[17] Nichizou Inc., "RCS Radio Frequency Control System, SPG Circuit Board, Hardware Specifications,' first revision (in Japanese), Osaka, 2004

[18] See http://www.scilab.org/; Institut National de Recherche en Informatique et en Automatique (INRIA), Domaine de Voluceau, BP 105, 78153 Le Chesnay Cedex, France.

[19] Nichizou Inc., "RCS Radio Frequency Control System, RFG Circuit Board, Hardware Specifications," first revision (in Japanese), Osaka, 2004.

[20] A. Schnase et al. (J-PARC Ring-RF Group), Review: Ring LLRF System feedback Simulations, KEK, Tsukuba, Japan, 2005.

[21] Nichizou Inc., "RCS Radio Frequency Control System, PFB Circuit Board, Hardware Specifications," first revision (in Japanese), Osaka, 2005.

[22] F. Blas and R. Garoby, in Proceedings of the Particle Accelerator Conference, San Francisco, CA, 1991 (IEEE, Piscataway, NJ, 1991), p. 1398. 